\title{
ARRDC3 suppresses breast cancer progression by negatively regulating integrin $\beta 4$
}

\author{
KM Draheim ${ }^{1}$, H-B Chen ${ }^{1}$, Q Tao ${ }^{2}$, N Moore ${ }^{1}$, M Roche $^{1}$ and S Lyle ${ }^{1}$ \\ ${ }^{1}$ Department of Cancer Biology, University of Massachusetts Medical School, Worcester, MA, USA and ${ }^{2}$ Beth Israel Deaconess \\ Medical Center, Boston, MA, USA
}

\begin{abstract}
Large-scale genetic analyses of human tumor samples have been used to identify novel oncogenes, tumor suppressors and prognostic factors, but the functions and molecular interactions of many individual genes have not been determined. In this study we examined the cellular effects and molecular mechanism of the arrestin family member, $A R R D C 3$, a gene preferentially lost in a subset of breast cancers. Oncomine data revealed that the expression of ARRDC3 decreases with tumor grade, metastases and recurrences. ARRDC3 overexpression represses cancer cell proliferation, migration, invasion, growth in soft agar and in vivo tumorigenicity, whereas downregulation of ARRCD3 has the opposite effects. Mechanistic studies showed that ARRDC3 functions in a novel regulatory pathway that controls the cell surface adhesion molecule, $\beta-4$ integrin (ITGß4), a protein associated with aggressive tumor behavior. Our data indicates ARRDC3 directly binds to a phosphorylated form of ITGß4 leading to its internalization, ubiquitination and ultimate degradation. The results identify the ARRCD3-ITGß4 pathway as a new therapeutic target in breast cancer and show the importance of connecting genetic arrays with mechanistic studies in the search for new treatments.
\end{abstract}

Oncogene (2010) 29, 5032-5047; doi:10.1038/onc.2010.250; published online 5 July 2010

Keywords: ARRDC3; breast cancer; integrin $\beta 4$; TLIMP

\section{Introduction}

The basal-like subset of breast cancer was first identified as tumors lacking hormone receptor and human epidermal growth factor receptor 2 amplification with a gene expression profile similar to basal or myoepithelial cells of the breast (Perou et al., 2000). Basal-like breast cancers account for $8-37 \%$ of all breast cancers (depending on the definition criteria) and is associated

Correspondence: Dr S Lyle, Department of Cancer Biology, University of Massachusetts Medical School, 364 Plantation Street-LRB 411, Worcester, MA 01605, USA.

E-mail: stephen.lyle@umassmed.edu

Received 18 December 2009; revised 10 April 2010; accepted 12 May 2010; published online 5 July 2010 with a poor prognosis; increased development of distant metastasis, decreased survival rate and increased mortality (reviewed in (Rakha et al., 2008; Voduc and Nielsen, 2008; Rakha and Ellis, 2009)). As these tumors lack the expression of estrogen and progesterone receptors as well as human epidermal growth factor receptor 2 , there are limited options that are largely ineffective in treating patients suffering from basal-like breast cancer.

Large-scale genetic analyses of human tumor samples have generated a wealth of molecular information and have identified potential tumor suppressors, oncogenes and prognostic factors (Perou et al., 2000; Sørlie et al., 2001; Sotiriou and Pusztai, 2009). The challenge now is to study the function of these new genes and understand their mechanisms of action to validate their clinical utility and confirm their potential use as targets of intervention. The arrestin family member $A R R D C 3$ is one such gene that was recently found within a cluster on chromosome 5 deleted in $17 \%$ of basal-like breast cancers (compared with $0 \%$ in luminal breast-cancers) suggesting a role as a tumor suppressor (Adelaide et al., 2007). ARRDC3 contains structural homology to the arrestin family of proteins, which has an important role in the internalization and subsequent regulation of $\mathrm{G}$ protein-coupled receptors (GPCRs). Although ARRDC3 has been classified as an $\alpha$-arrestin, there is a cluster of acidic and hydrophobic residues that may bind to clathrin, suggesting a role in membrane protein internalization (Alvarez, 2008). This is further supported by the putative structural homology between ARRDC3 and other arrestin family members VPS26 and $\beta$-arrestin (Aubry et al., 2009). A role for ARRDC3 connecting the PPAR $\gamma$ signal and endosomal functions has been suggested (Oka et al., 2006).

Integrins (ITGs) are cell surface adhesion molecules which mediate cell-extracellular matrix and cell-cell interactions. Binding of ITGs to their ligands initiates several signaling events that modulate many cellular behaviors, such as adhesion, proliferation, survival, motility, gene expression and differentiation (Dowling et al., 1996; Fuchs et al., 1997; Hynes, 2002; Watt, 2002; Arnaout et al., 2005; Lipscomb et al., 2005; Wilhelmsen et al., 2006; Germain et al., 2009; Vicente-Manzanares et al., 2009). The ITG $\beta 4$ subunit was initially identified in cancer as a tumor-related antigen associated with metastasis (Falcioni et al., 1989; Lee et al., 1992) and was later found to promote motility and invasion in 
carcinoma cells (Rabinovitz and Mercurio, 1996; O'Connor et al., 1998; Nikolopoulos et al., 2004). ITG $\beta 4$ signaling increases invasive potential and has been shown to sustain the survival of carcinoma cells in stressful environments (Yoon et al., 2005; Baril et al., 2007; Chen et al., 2009). Recent human studies have found that ITG $\beta 4$ expression is correlated to breast cancer size and grade (Diaz et al., 2005), and significantly correlates with basal-like breast cancer (Lu et al., 2008). ITG $\beta 4$ expression is also linked to poor patient prognosis in a variety of cancers (Raymond et al., 2007).

Despite its significance in tumor progression, surprisingly little is known about the regulation of ITG $\beta 4$ at the protein level. It is phosphorylated during signal transduction and thought to be internalized, trafficked around the cell, and either recycled or degraded (Hemler, 2001; Caswell and Norman, 2006, 2008; Wilhelmsen et al., 2007; Dutta and Shaw, 2008; Germain et al., 2009). It has been hypothesized that after hemidesmosome disrupting signals, newly liberated ITG $\beta 4$ is rendered capable for signaling. Activation results in the release of ITG $\beta 4$ from interactions with the cytokeratin cytoskeleton and allows for de novo interaction with the actin cytoskeleton and signaling molecules (Rabinovitz and Mercurio, 1996; Yoon et al., 2005; Mitra and Schlaepfer, 2006). However, little is known about the mechanism for which this occurs.

To understand the function of ARRDC 3 we used overexpression and short hairpin RNA-mediated downregulation in human breast cancer cells. We showed significant effects on breast cancer cell migration and growth that coincided with dramatic effects on the cell surface protein ITG $\beta 4$. In this study we showed that ARRDC3 functions as a novel regulator of tumor progression in breast cancer because of its effects on ITG $\beta 4$ internalization and degradation.

\section{Results}

\section{ARRDC3 expression is downregulated during tumor progression}

To investigate how ARRDC3 expression is altered during carcinogenesis, we used Oncomine to analyze previously published microarray data. Levels of $A R R D C 3$ mRNA are lower in breast cancer tissues when

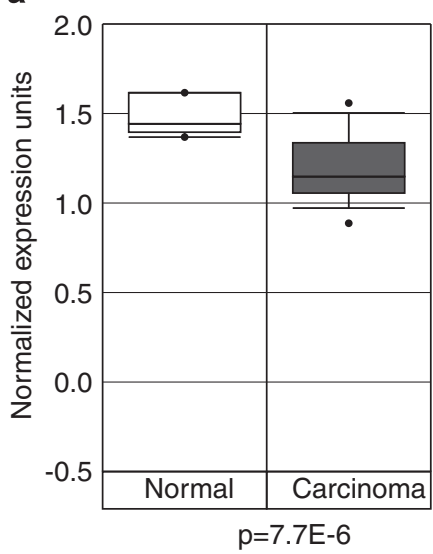

b

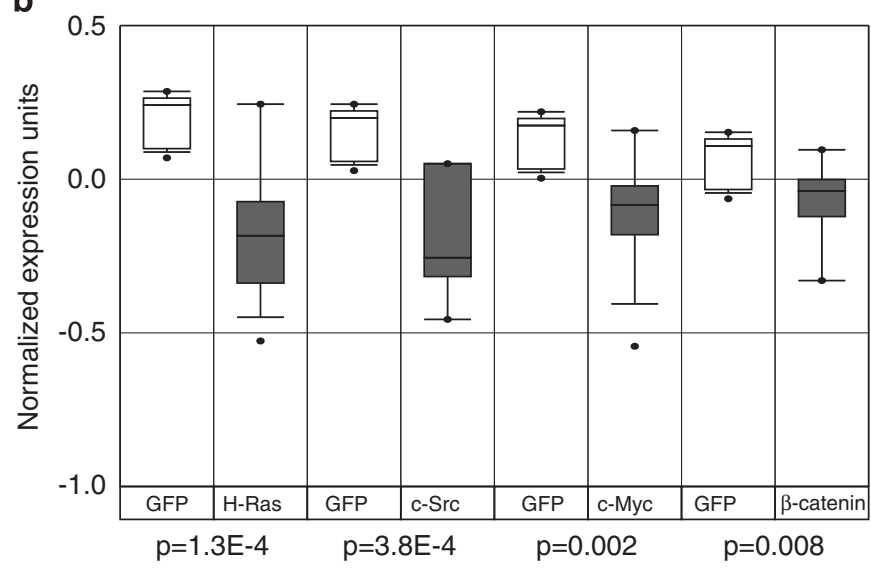

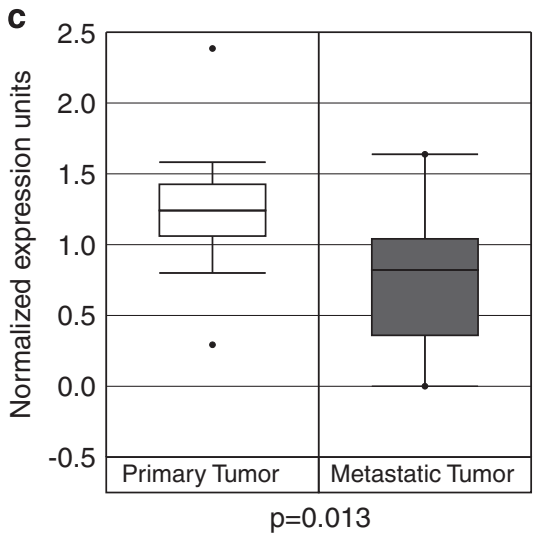

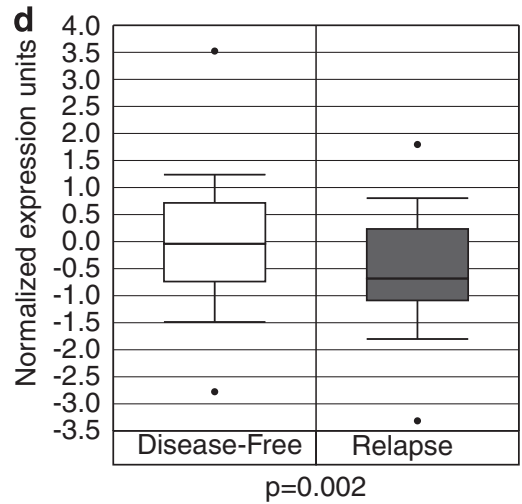

Figure 1 Downregulation of ARRDC3 is an early event in carcinogenesis. Oncomine was used to analyze previously published microarray data. (a) Levels of $A R R D C 3 \mathrm{mRNA}$ are decreased in human breast cancers when compared with normal breast tissue. (b) ARRDC3 mRNA levels decrease in human mammary epithelial cells after transformation with various oncogenes. (c) Levels of $A R R D C 3 \mathrm{mRNA}$ are decreased in metastatic lesions when compared with the primary tumor in the same patient. (d) Expression of $A R R D C 3 \mathrm{mRNA}$ is decreased in the initial ER + tumors from patients that have relapsed when compared with 5-year disease-free patients. All data, including $P$-values, were calculated from Oncomine. 
compared with normal tissue (Figure 1a). Furthermore, $A R R D C 3$ mRNA levels decrease on the transformation of human mammary epithelial cells (Figure 1b). Oncomine data also revealed a decrease in $A R R D C 3$ mRNA in metastatic tumors when compared with the primary tumor (Figure 1c). In addition, $A R R D C 3$ levels are lower in tumors of breast cancer patients that relapsed within 5 years of diagnosis when compared with patients that remained disease free for $>5$ years (Figure 1d). Combined, these data indicate that ARRDC3 repression occurs early in carcinogenesis, decreases throughout tumor progression and suggests that low ARRDC3 may indicate a poor prognosis.

\section{ARRDC3 affects in vitro cancer cell tumorigenicity}

To evaluate the potential role of ARRDC3 as a suppressor of tumor growth, we generated stable cancer cell lines either overexpressing or repressing ARRDC3 in the basal-like breast cancer cell line MDA-MB-231 cells. The MDA-MB-231 line was used because it is considered a basal-like breast cancer cell line that had detectable levels of ARRDC3 and ITG $\beta 4$ when compared with other cell lines (Supplementary Figure S1). In Addition, MDA-MB-231 cells depend on ITG $\beta 4$ signaling for cell adhesion, survival, migration and invasion (Lipscomb et al., 2003, 2005). Expression of ARRDC3 indicated that there was not a chromosomal 5 deletion therefore, we could manipulate levels to observe effects on tumorigenicity.

We first noticed a change in the comparative growth rates. ARRDC3 overexpression causes a decrease in cell growth rates, whereas the repression of ARRDC3 increases cell growth (Figure 2a). To investigate the effects on invasiveness, sublines were used in a chemoinvasion assay. Overexpression of ARRDC3 caused a $50 \%$ reduction in the number of invasive cells, wheras repression of ARRDC3 caused a twofold increase (Figure 2b). Sublines were then used in a wound assay to test whether ARRDC3 affects cancer cell migration. We found a dramatic decrease in cell migration when ARRDC3 was overexpressed (Figure 2c). Sublines with repressed ARRDC3 exhibited a significant increase in migration rate. We next evaluated the importance of ARRDC3 for anchorage independent growth by calculating the colony number and size after 4-weeks growth in soft agar. There was a significant decrease in colony number in the ARRDC3 overexpressing sublines, whereas sublines with repressed ARRDC3 showed a significant increase in colony number (Figure 2d). ARRDC3 expression also affected the size of the colonies; sublines with repressed ARRDC3 had a higher percentage of colonies larger than $>200 \mu \mathrm{m}$, whereas lines overexpressing ARRDC3 had a lower percentage of colonies $>200 \mu \mathrm{m}$ (Figure 2e).

\section{ARRDC3 suppresses in vivo tumorigenicity}

To assess the role of ARRDC3 in tumor growth in vivo, the stable subclones of MDA-MB-231 cells were injected into the mammary fat pad of nude mice $(n=20$ for each subline). All subclones formed tumors efficiently in $85-100 \%$ of the mice suggesting that ARRDC3 does not affect in vivo tumor incidence. However, sublines with repressed ARRDC3 formed measurable tumors more quickly ( 2 weeks) when compared with the control line (3 weeks). In contrast, the formation of tumors from the ARRDC3 overexpressing line was delayed (4 weeks) when compared with the control line ( 3 weeks). After 7 weeks of injection, mice were killed and tumors were dissected and measured. ARRDC3 significantly suppressed tumor growth in vivo as determined by the final tumor volume measurements (Figures $3 \mathrm{a}-\mathrm{c}$ ). All tumors showed a similar undifferentiated solid tumor morphology (Supplementary Figure S2a). To determine whether there was a difference in the proliferation, sections from the xenograft tumors $(n=5$ tumors for each cell line) were stained for $\mathrm{Ki} 67$. As expected from the in vitro studies, repressing ARRDC3 increases the percent of Ki67 positive cells by almost twofold, whereas overexpressing ARRDC3 decreases the number of Ki67positive cells by approximately threefold (Figure $3 \mathrm{~d}$ and Supplementary Figure S2b).

To assess the contribution of ARRDC3 to in vivo tumor cell survival, sections of xenograft tumors were evaluated for central necrosis. The areas of necrosis and total tumor area were quantified using ImageJ software ( $n=4$ tumors of each cell line). Although larger tumors are generally more necrotic, tumors derived from the shARRDC3 lines contained significantly less necrosis when compared with control tumors (Figures $4 \mathrm{a}$ and $\mathrm{b}$ ). In contrast, tumors from the ARRDC3 overexpressing cell line, although smaller, were highly necrotic (Figures 4a and b). Terminal deoxynucleotidyl transferase-mediated dUTP-biotin nick end labeling staining was also carried out on sections from the xenograft tumors. The expression of ARRDC3 seemed to have no effect on the number of apoptotic cells (Figure 4c and Supplementary Figure S2c), suggesting that ARRDC3 functions independently from the apoptotic pathway.

Figure 2 Expression of ARRDC3 affects in vitro tumorigenicity Each line was tested in triplicate and data bars represent mean \pm s.e.m. Single asterisk represents $P<0.05$, whereas a double asterisk represents $P<0.001$ as determined by Student's $t$-test. (a) Growth curves from the stable lines show that ARRDC3 overexpression suppresses cellular proliferation, whereas repression enhanced proliferation. (b) Matrigel chemo-invasion assay show that ARRDC3 overexpression suppresses invasion, whereas repression enhances invasion. (c) Wound assay of mitomycin-C treated cells shows that overexpression of ARRDC3 leads to a decrease in cell migration, whereas RNA interference-mediated repression of ARRDC3 increases cell migration. (d) ARRDC3 overexpression suppresses anchorage independent growth, whereas repression enhances anchorage independent growth. (e) Both colony number and colony size are affected. Dark gray bars represent total colonies (at least $50 \mu \mathrm{m}$ ), whereas light gray bars represent colonies larger than $200 \mu \mathrm{m}$. 
ARRDC3 negatively regulates ITG $\beta 4$

Initial studies using adenoviral vectors to overexpress ARRDC3 gave approximately 40 -fold increase in protein and caused a complete detachment of MDA-
MB-231 cells from the substratum after 1 week in culture suggesting a defect in cell adhesion. As cell adhesion and tumorigenic properties of MDA-MB-231 cells are dependent on ITG $\beta 4$, we hypothesized that
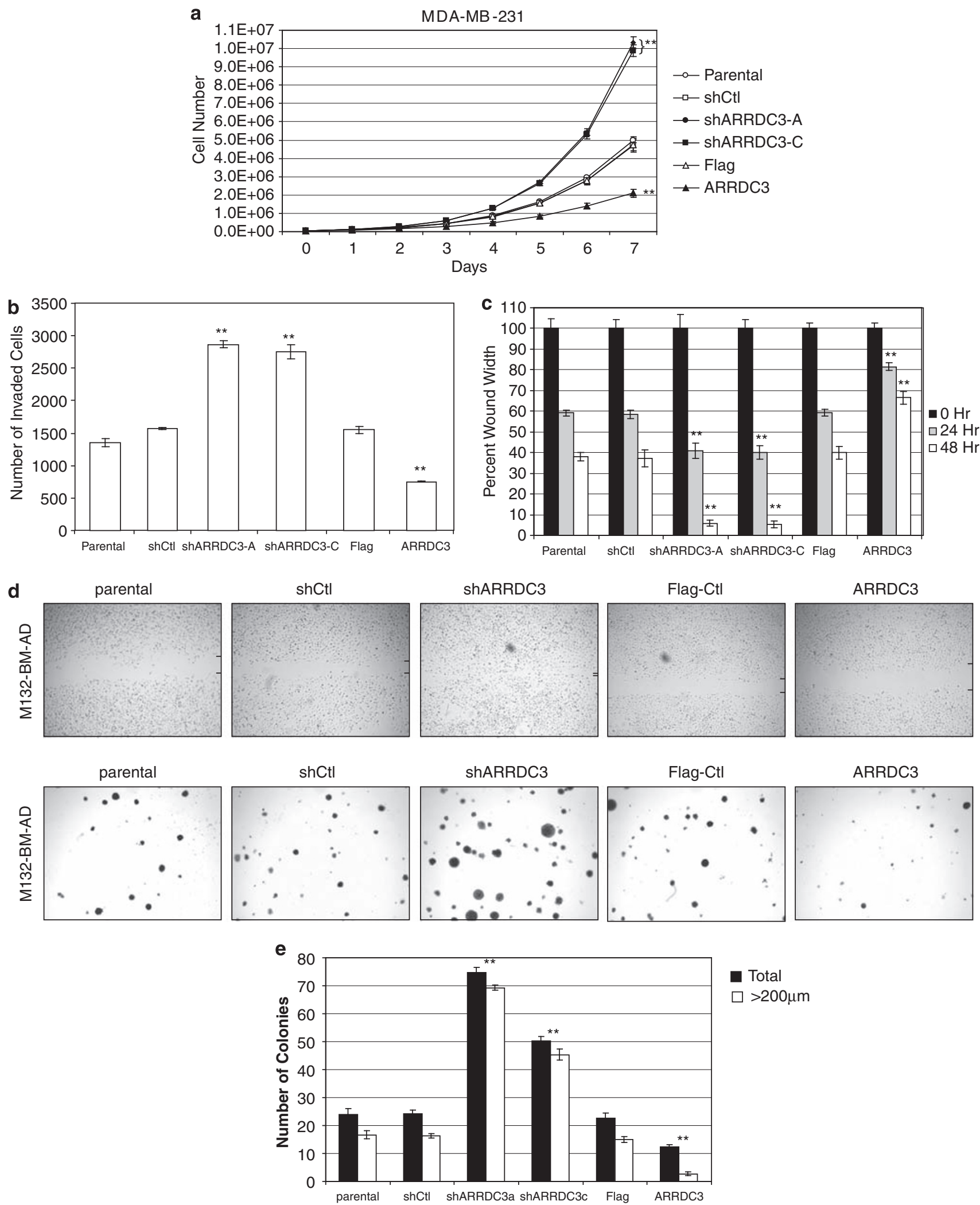

Total $\square>200 \mu \mathrm{m}$ 
ARRDC3 was affecting ITG $\beta 4$ expression. To determine whether ARRDC3 expression influences ITG $\beta 4$ levels in breast cancer cells, the stable lines with approximately fivefold ARRDC3 overexpression and RNA interference knockdown were analyzed by western blot. Overexpression of ARRDC3 caused a significant decrease in ITG $\beta 4$ protein levels, whereas cells with repressed ARRDC3 expression show a marked increase in ITG $\beta 4$ (Figure 5a). To verify ITG $\beta 4$ was only affected in cells with altered ARRDC3, cells were

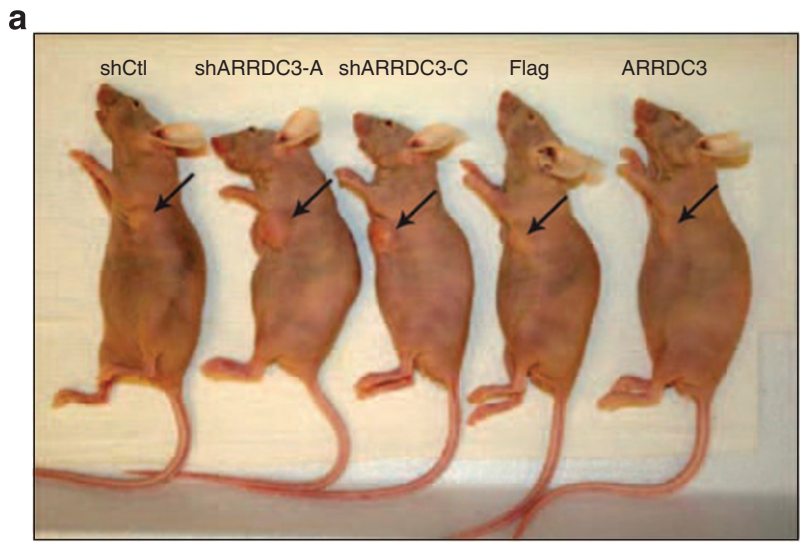

b
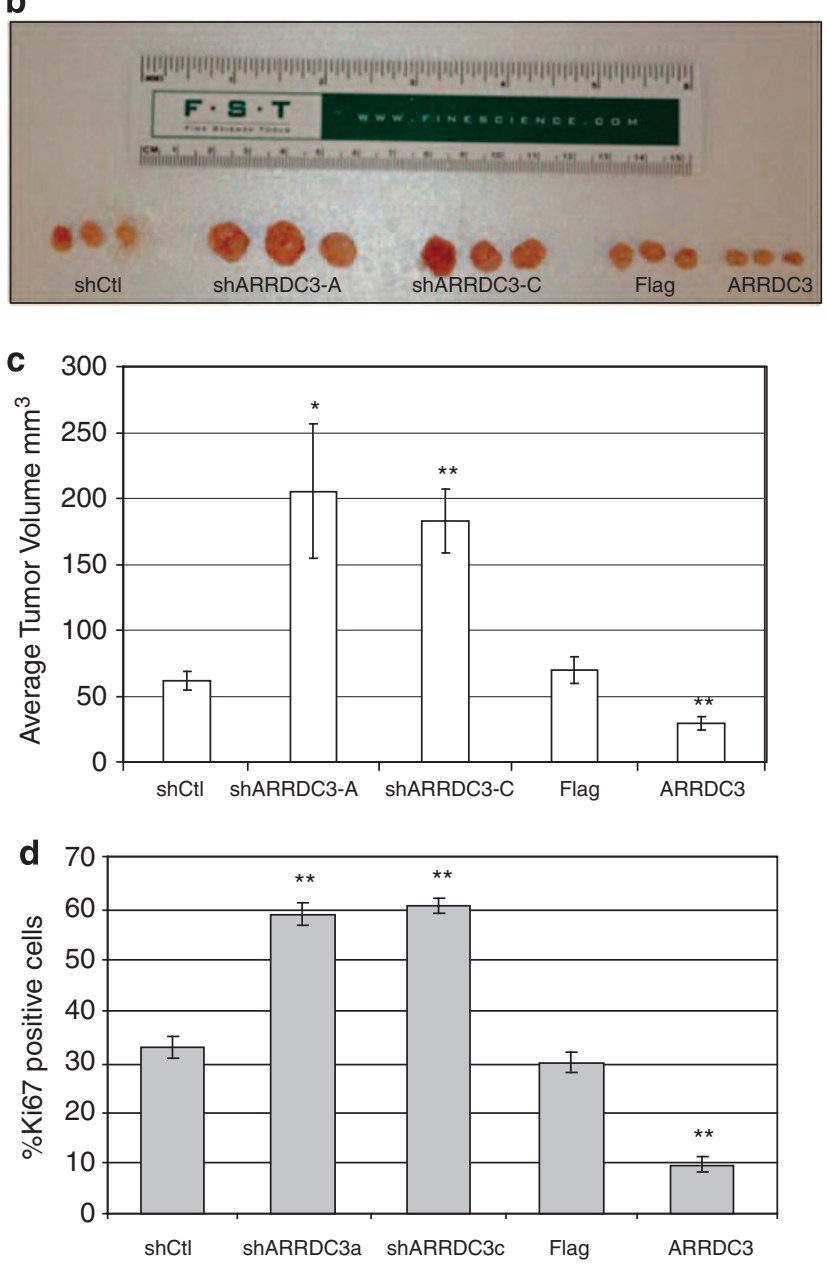

infected with a green fluorescent protein-expressing ARRDC3 adenovirus and evaluated for ITG $\beta 4$ levels using immunofluorescence. Cells that overexpress ARRDC3 showed significant downregulation of ITG $\beta 4$ surface levels, whereras adjacent uninfected cells showed no change in ITG $\beta 4$ protein levels (Figure 5b). Additionally, a partial detachment of ARRDC3 overexpressing cells from the substratum can be seen in culture $24 \mathrm{~h}$ after infection (Figure 5b). As expected, ITG $\beta 4$ expression and cell attachment were not affected by infection with the control green fluorescent protein adenovirus. Flow cytometric analysis also showed that overexpression of ARRDC3 causes an ablation of ITG $\beta 4$ protein from the cell surface (Figure 6c).

Interestingly, although ITG $\beta 4$ is altered in our stable MDA-231 cell lines (Figure 5a), these cells did not show the loss of adhesion seen in adenoviral infected cells. This is likely because of the higher expression levels that can be achieved with adenovirus.

\section{ARRDC3 regulates ITG $\beta 4$ protein levels in a proteosome-dependent manner}

We next wanted to determine the mechanism of ITG $\beta 4$ degradation in breast cancer cells. Parental MDA-MB231 cells were infected with adARRDC3 and then treated with the proteosome inhibitor lactacystin for $6 \mathrm{~h}$. The addition of $25 \mu \mathrm{M}$ lactacystin prevented the ARRDC3mediated decrease in ITG $\beta 4$ protein levels as determined by immunofluorescence and western blots (Figures 6a and b). Lactacystin treatment did not prevent internalization of surface ITG $\beta 4$, although a low level of ITG $\beta 4$ was still detectable on the surface (Figure 6c), suggesting that undegraded protein can be recycled to the cell surface. To exclude the possibility of lysosomal contribution to the protein degradation, ARRDC3 overexpressing cells were treated with the lysosomal inhibitor, chloroquine at $50 \mu \mathrm{M}$ for $18 \mathrm{~h}$. Chloroquine treatment did not prevent ITG $\beta 4$ reduction after infection with adARRDC3 (Supplementary Figures S3a and b).

As the regulation of ITG $\beta 4$ by ARRDC3 seemed to be dependent on the proteosome, and as to other arrestin family members have a role in targeting receptors for degradation (Gurevich and Gurevich, 2006; Bhandari et al., 2007; Kendall and Luttrell, 2009; Zhang et al., 2009), we asked whether ARRDC3

Figure 3 ARRDC3 negatively regulates in vivo tumorgenicity. Stable cells lines of MDA-MB-231 cells were injected into the mammary fat pads of immunocompromised mice $(n=20$ injections for each cell line) and grown for 7 weeks. (a-b) Overexpression of ARRDC3 (ARRDC3) led to a decrease in in vivo tumor size compared with vector control (Flag), whereas repression of ARRDC3 (shARRDC3) led to an increase in tumor size compared with control (shCtl). (c) When quantified, the differences in final tumor volume were statistically significant compared with scrambled short hairpin RNA and empty vector controls. (d) ARRDC3 affects the in vivo proliferation of tumor cells as determined by percentage of Ki67positive cells. Xenograft tumor sections were analyzed for Ki67 using IHC. Over 1500 cells per tumor were counted and scored either $\mathrm{Ki} 67$ + or $\mathrm{Ki} 67-$. In all, four to five tumor samples per group were analyzed and data bars represent mean \pm s.e.m. Single asterisk represents $P<0.05$, whereas a double asterisk represents $P<0.001$ as determined by Student's $t$-test. 
a

shCtl

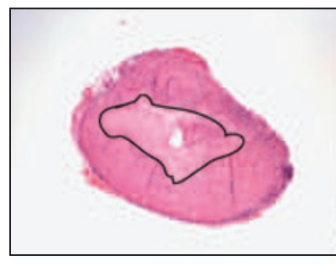

ShARRDC3-A

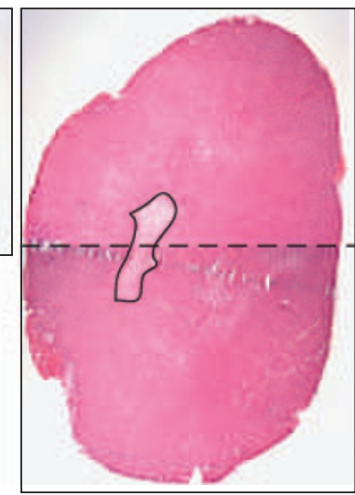

b

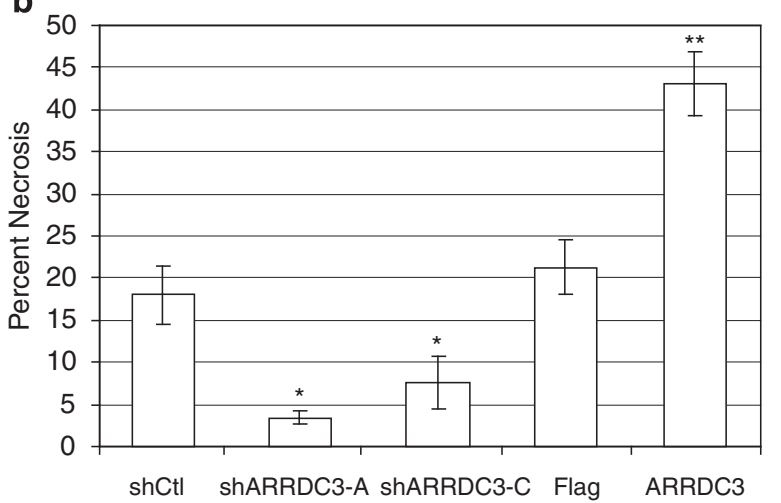

ShARRDC3-C

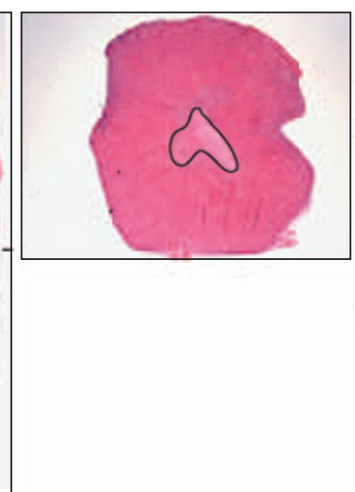

Flag

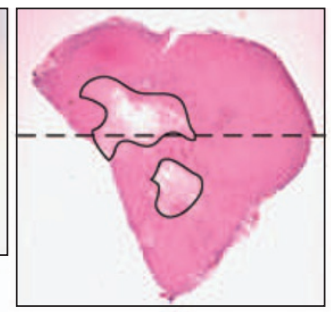

ARRDC3

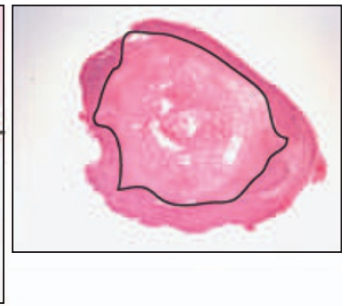

C

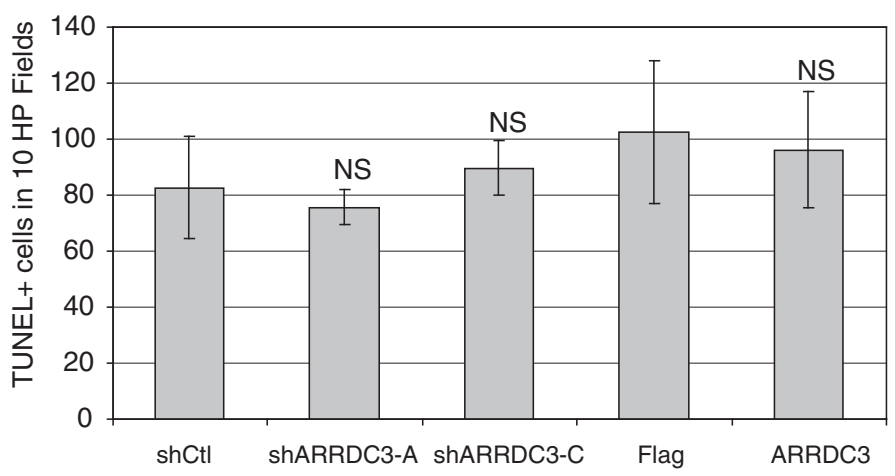

Figure 4 ARRDC3 repression promotes in vivo cell survival. Tumors were cut in half along the longest axis therefore the center-most section of the tumor was used to make slides. Changes in necrosis are more evident when tumors of comparable size are analyzed. (a, b) Repression of ARRDC3 (shARRDC3) leads to a decrease in xenograft tumor necrosis (outlined in black) compared with control (shCtl), whereas overexpression of ARRDC3 (ARRDC3) leads to an increase in xenograft tumor necrosis compared with vector control (Flag). Dashed line represents the point where two images were merged to visualize the entire tumor section. Five tumors from each line were analyzed and the area of necrosis measured. Data bars represent mean \pm s.e.m. Single asterisk represents $P<0.05$, whereas a double asterisk represents $P<0.001$ as determined by Student's $t$-test. (c) ARRDC3 does not affect the number of apoptotic cells in xenograft tumors. Terminal deoxynucleotidyl transferase-mediated dUTP-biotin nick end labeling (TUNEL) analysis was performed on tumor sections and the number of positive cells in 10 high-powered fields $(\times 40)$ was counted. Five tumors per group were analyzed and data bars represent mean \pm s.e.m. NS, not significant.

interacts with ITG $\beta 4$ after it is targeted for degradation. Untreated MDA-MB-231 cells show an undetectable amount of ubiquitinated ITG $\beta 4$, even after immunoprecipitation. However, when cells were pretreated with proteosome inhibitor lactacystin, higher molecular weight bands of ITG $\beta 4$ were detected after immunoprecipitation with both ARRDC3 and ITG $\beta 4$ antibodies (Figure 6d). Immunoblotting for Ubiquitin revealed that almost equal amounts of ubiquitinatedITG $\beta 4$ is coimmunoprecipitated with the ARRDC3 antibody when compared with the ITG $\beta 4$ immunoprecipitation (Figure 6d). Thus, ARRDC3 binds ITG $\beta 4$ after ubiquitination, and seems likely to have a role in targeting ITG $\beta 4$ for degradation.

\section{ARRDC3 directly interacts with activated ITG 34}

A novel phosphorylation site on ITG $\beta 4$, serine-1424, was recently identified as important in hemidesmosome disassembly and was enriched on the trailing edge of migrating cells (Germain et al., 2009). This phospho- rylation, along with the phosphorylation of other serines, results in the disassembly of the hemidesmosome and mobilization of ITG $\beta 4$ to actin-rich protrusions (Rabinovitz et al., 2004; Wilhelmsen et al., 2007; Germain et al., 2009). To determine whether this site is important in ARRDC3-mediated ITG $\beta 4$ internalization, endogenous expression patterns of ITG $\beta 4$, ITG $\beta 4$ pS1424 and ARRDC3 were examined using confocal microscopy on migrating cancer cells. ARRDC3 colocalized with ITG $\beta 4$ only on the lagging edge of the cell, where ITG $34-p S 1424$ is enriched (Figure 7a). We determined that ARRDC3 physically interacts with ITG 34 -pS1424 by endogenous coimmunoprecipitation (Figure 7b). Densitometric analysis show approximately $70 \%$ of ITG $\beta 4-p S 1424$ is coimmunoprecipitated by ARRDC3 antibody when compared with ITG $\beta 4$ antibody. The densitometric ratio of precipitated pS1424/ ITG 34 is 3.5-fold higher for the ARRDC3 coimmunoprecipitation when compared with the ITG $\beta 4$ coimmunoprecipitation suggesting that ARRDC3 preferentially interacts with this activated form of ITG $\beta 4$. 

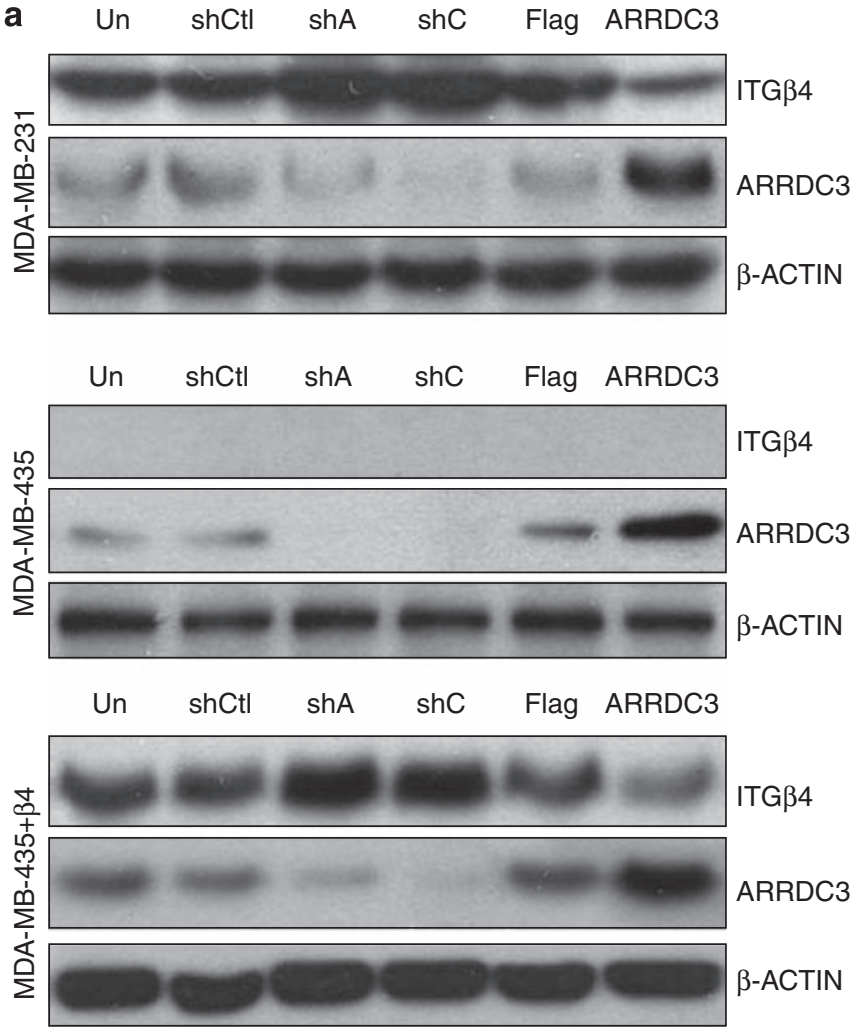

b
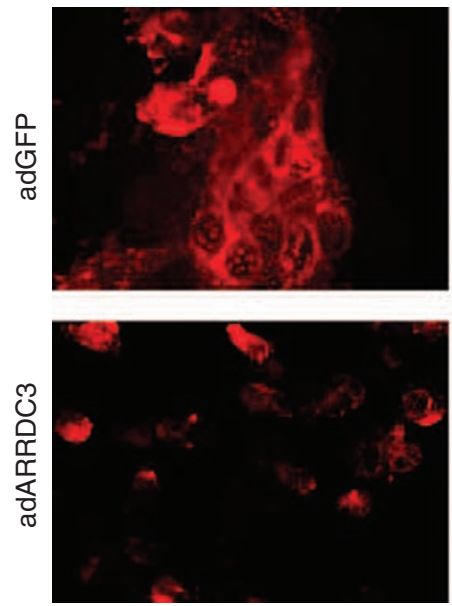

GFP
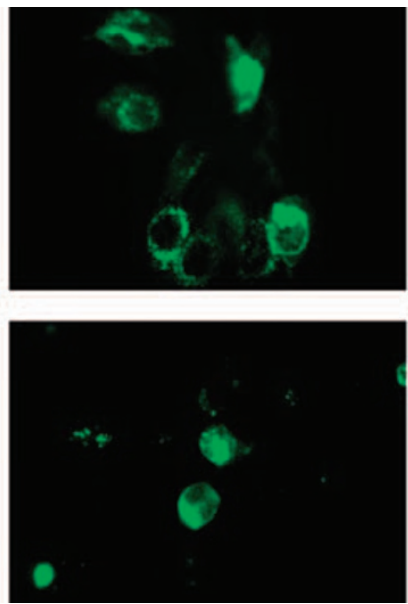

DAPI
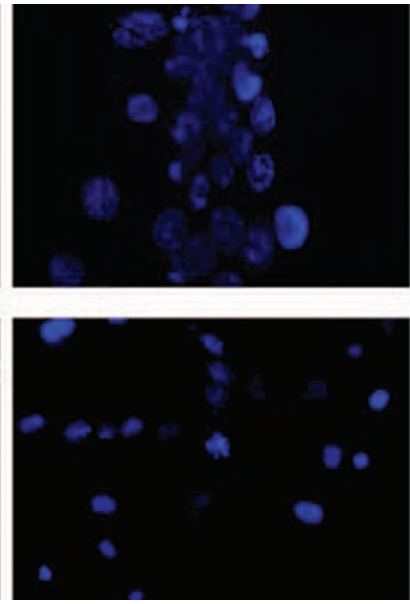

Merge
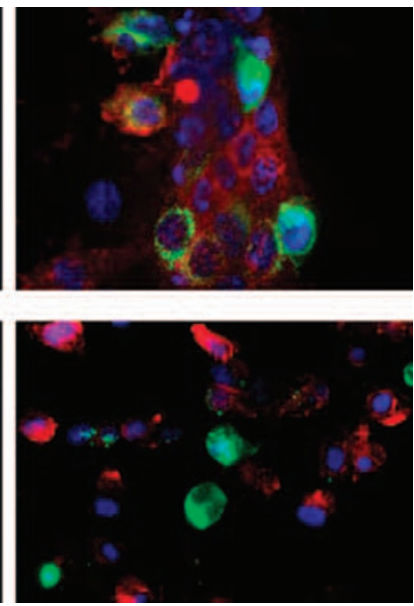

Figure 5 ARRDC3 negatively regulates ITG 34 . (a) Western blot analysis shows that ITG $\beta 4$ protein level is affected by ARRDC3 expression. Stable alterations of ARRDC3 expression in MDA-MB-231, MDA-MB-435 and MDA-MB-435 + $\beta 4$ cell lines. When ARRDC3 expression was repressed using two separate short hairpin RNA constructs (shA or shC), cells showed an increase in ITG $\beta 4$ protein when compared with control cells (Un or shCtl). Conversely, when cells were made to overexpress ARRDC3 (ARRDC3), ITG $\beta 4$ levels decreased when compared with vector control cells (Flag) or untransfected parental cells (Un). (b) Immunofluorescence shows that when MDA-MB231 cells overexpress ARRDC3 using a green fluorescent protein (GFP)-expressing adenovirus (adARRDC3), ITG $\beta 4$ levels decrease when compared with uninfected cells (no GFP expression) and cells infected with a control GFP-expressing adenovirus (adGFP).

Figure 6 In breast cancer cells, ARRDC3 directly interacts with ubiquitinated ITG $\beta 4$ and negatively regulates protein levels in a mechanism dependent on the proteosome. (a, b) Cells can maintain high levels of ITG 34 after ARRDC3 overexpression if treated with proteosome inhibitor Lactacystin for $6 \mathrm{~h}$. (a) Western blot analysis shows that ITG $\beta 4$ levels after ARRDC3 overexpression is restored when the proteosome is inhibited. Inhibition of the proteosome is demonstrated by the accumulation of $\mathrm{I} \kappa \mathrm{B} \alpha$. (b) Immunofluorescence demonstrates that ITG $\beta 4$ levels are retained after ARRDC3 overexpression if cells are treated with proteosome inhibitor. Cells positive for adARRDC3 (green fluorescent protein $(\mathrm{GFP}+))$ are outlined with a dashed line. (c) ARRDC3 causes a complete removal of ITG $\beta 4$ from the cell surface that is partially rescued with proteosome inhibition. The cell surface of live cells were then stained with an ITG $\beta 4$ antibody and analyzed by flow cytometry. Uninfected GFPnegative cells were gated out before ITG $\beta 4$ levels were examined. (d) Pretreatment of MDA-MB-231 cells with proteosome inhibitor lactacystin before the endogenous coimmunoprecipitation with antibodies against ARRDC3 and ITG $\beta 4$ enriches ubiquitinated forms of ITG $\beta 4$. Bands seen in the ubiquitin immunoblots were identical in size to ITG $\beta 4$ bands. Dotted line indicates noncontiguous lanes from the same film. 
ARRDC3 regulates breast cancer through ITG $\beta 4$

ARRDC3 specifically targets ITG $\beta 4$

Cancer cells frequently have defects within vesicular trafficking and/or endocytic pathways (Mosesson et al., 2008). To demonstrate that ARRDC3 specifically targets ITG $\beta 4$, rather than stimulating generalized endocytosis, we examined several cell surface proteins after cells were infected with either control or ARRDC3 over-expressing adenovirus. Analysis of nonpermeabi-

a

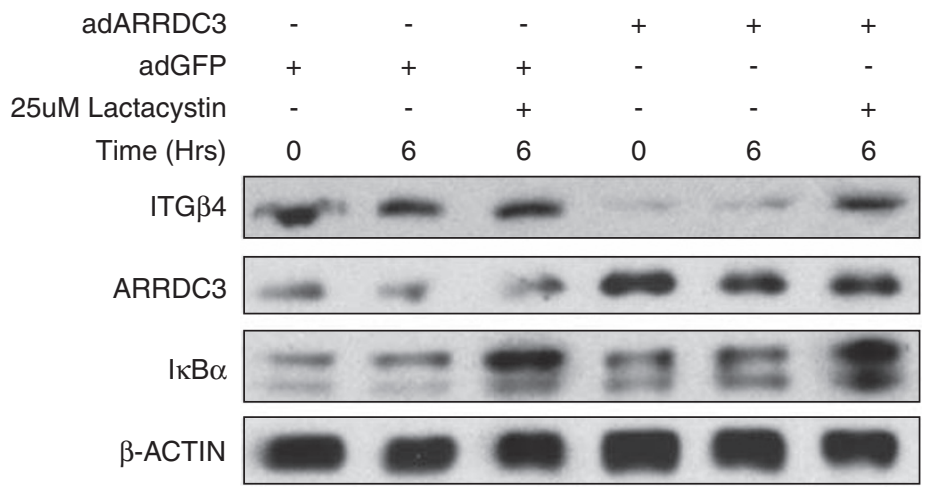

b
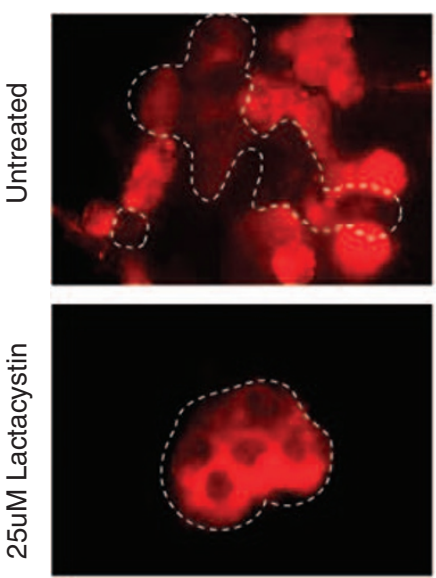

C
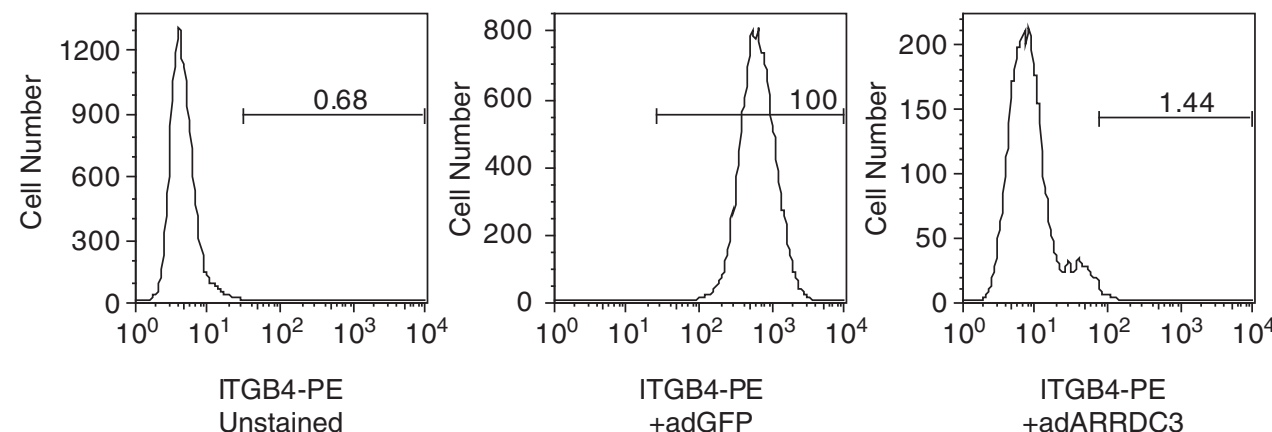

d

untreated

\begin{tabular}{ccc}
\hline \multicolumn{3}{c}{ IP } \\
\hline mIgG & ARRDC3 & ITG $\beta 4$
\end{tabular}

\begin{tabular}{lll}
\hline \multicolumn{3}{c}{25 uM Lactacystin } \\
\hline IP & \\
\hline mlgG & ARRDC3 & ITG 34 \\
\hline
\end{tabular}

IB: ITG $\beta 4$

$\frac{250 \mathrm{kDa}}{150 \mathrm{kDa}}$
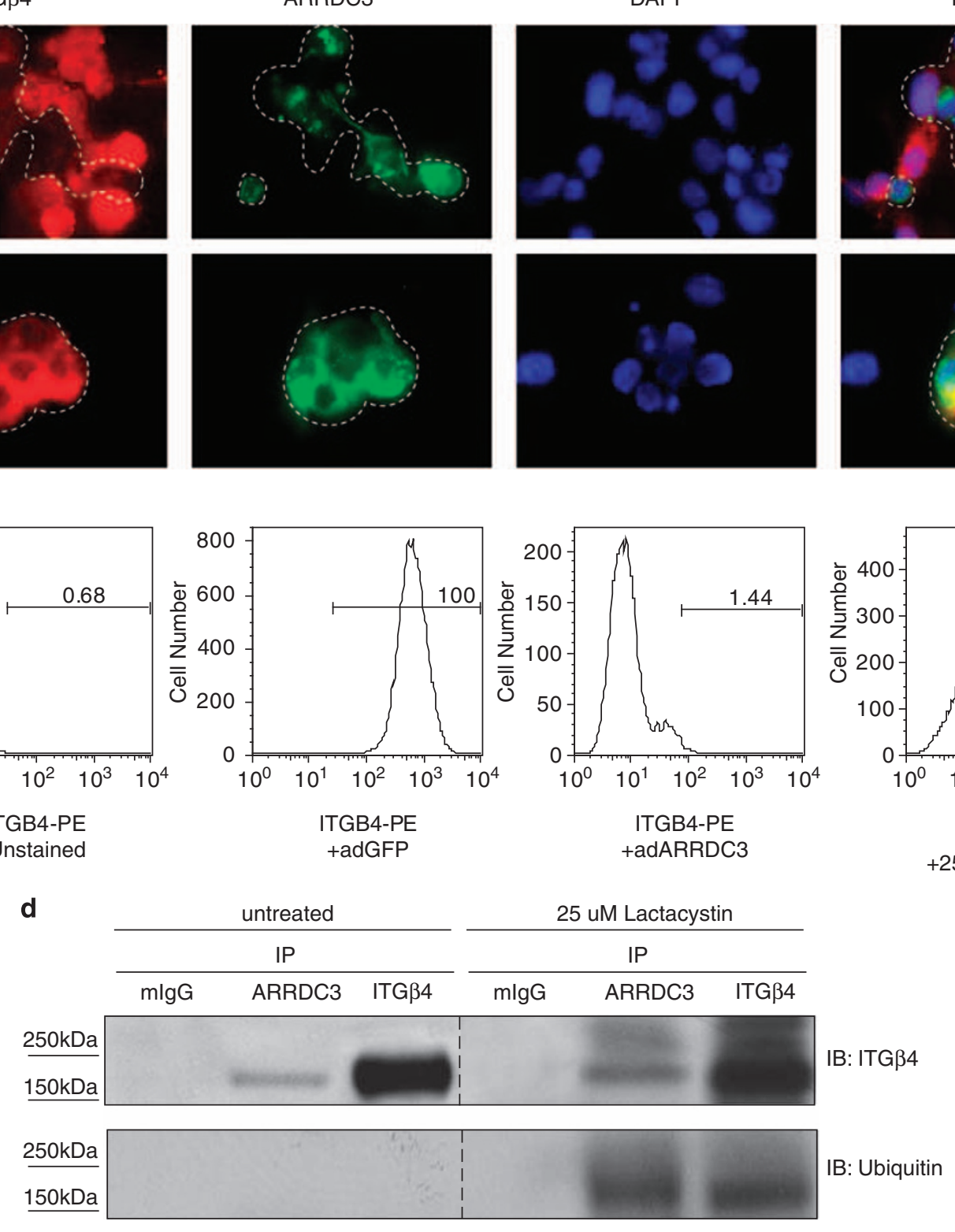
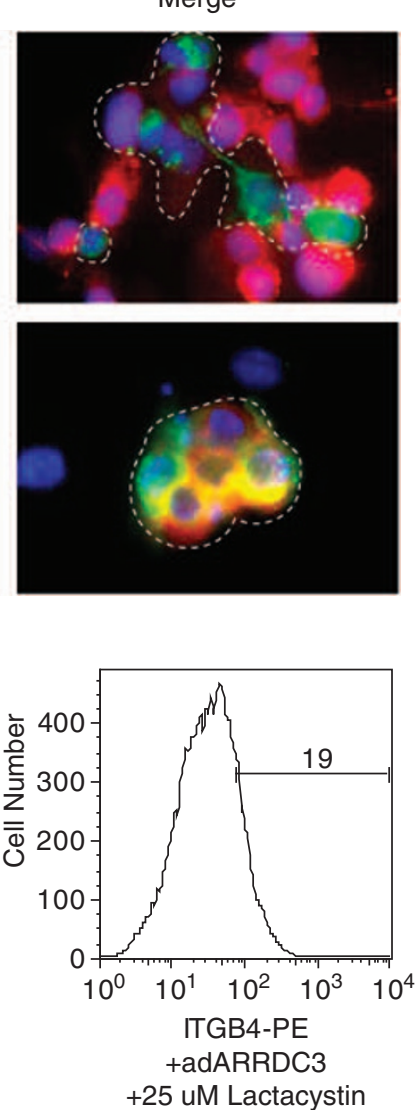
a

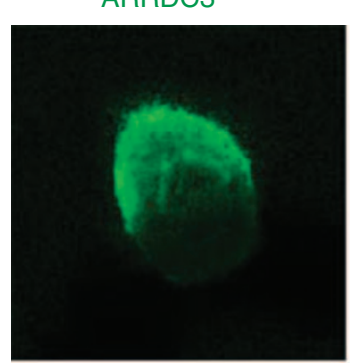

ARRDC3/ITGß4

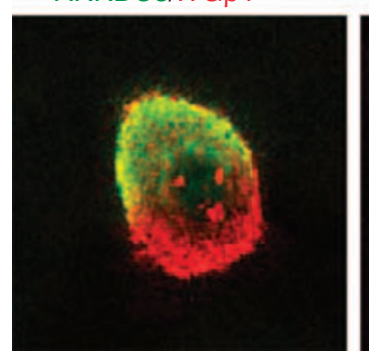

ITGß4

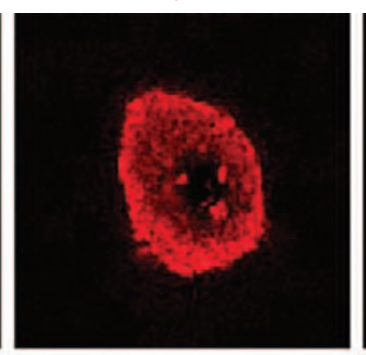

ITGß4/pS1424

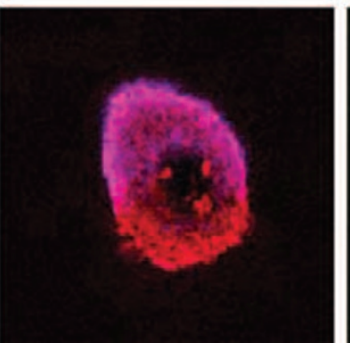

pS1424

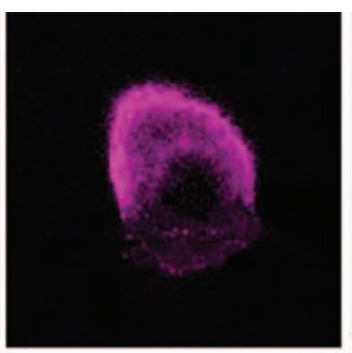

ARRDC3/pS1424

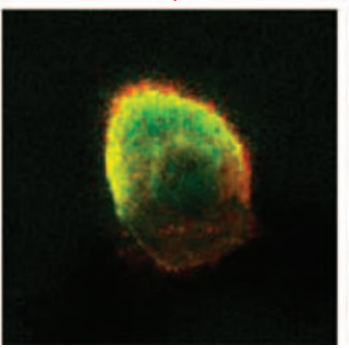

DAPI

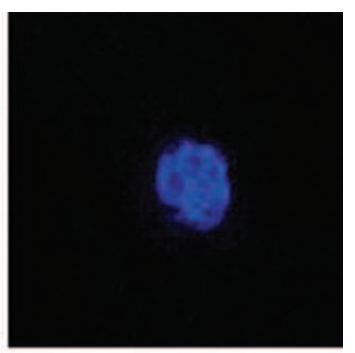

Brightfield

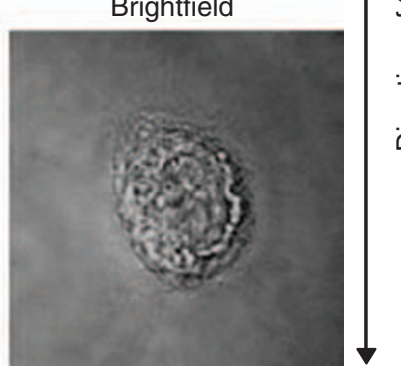

b IP

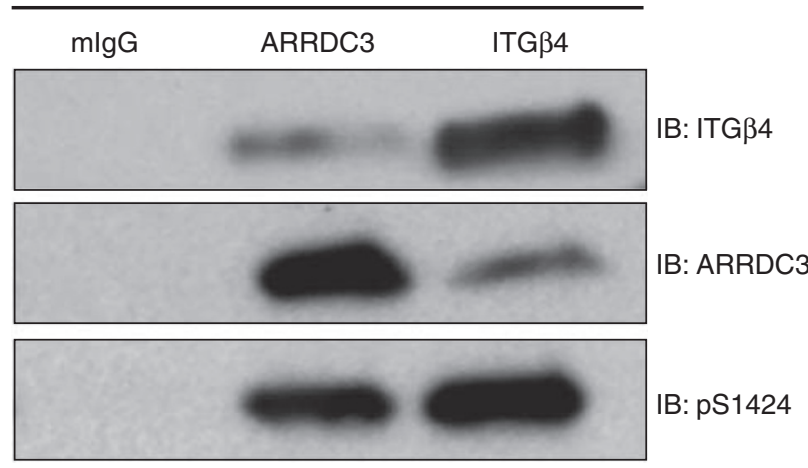

Figure 7 ARRDC3 preferentially interacts with ITG $\beta 4$ when phosphorylated on serine-1494. (a) Confocal images of a migrating MDA-MB-231 cell after wounding. Endogenous expression of ARRDC3, ITG $\beta 4$ and ITG $\beta 4$-pS1424 was detected. ARRDC3 colocalizes with ITG $\beta 4$ on the lagging edge of the cell, where ITG $\beta 4-p S 1424$ is located. (b) MDA-MB-231 cells were treated with phosphatase inhibitors $1 \mu \mathrm{M}$ sodium orthovanadate and $1 \mu \mathrm{M}$ sodium fluoride for $3 \mathrm{~h}$. Cell lysates were used in endogenous coimmunoprecipitation with antibodies against ARRDC3 and ITG $\beta 4$. ARRDC3 enriches ITG $\beta 4-p S 1424$ when compared with total ITG $\beta 4$. Bands seen in the phospho-S1424 immunoblots were identical in size to ITG $\beta 4$ bands.

lized cells by flow cytometry shows that ARRDC3 overexpression did not affect surface levels of ITG $\beta 1$, CD44 and EpCam (Figure 8).

\section{Effects of ARRDC3 on in vitro tumorigenicity \\ is dependent on ITG $\beta 4$}

To determine whether ITG $\beta 4$ is required to mediate the effects of ARRDC3 on cancer cell behavior, we used MDA-MB-435 (a cancer line which does not express ITG $\beta 4$ ) and MDA-MB-435 + $\beta 4$ cells (a daughter cell line engineered to overexpress ITG $\beta 4$ ), to create lines with altered ARRDC3 levels and assayed for tumorigenicity (Figure 5a).

Similar to MDA-MB-231 cells, the MDA-MB$435+\beta 4$ cells showed marked changes in proliferation, migration, invasion and anchorage independent growth.
Overexpression of ARRDC3 in MDA-MB-435+ $\beta 4$ cells caused decreased proliferation and migration, whereas repression of ARRDC3 lead to increased proliferation and migration (Supplementary Figures $\mathrm{S} 4 \mathrm{a}$ and $\mathrm{b}$ ). Cell invasion assays showed a 50\% decrease in the number of invasive cells in ARRDC3 overexpressing cells, whereas repression of ARRDC3 caused a twofold increase (Supplementary Figure S4c). Soft agar assays again showed that overexpression of ARRDC3 inhibits anchorage independent growth, whereas repressed ARRDC3 promotes in vitro tumorigenicity in the MDA-MB- $435+\beta 4$ cells. In contrast to the ITG $\beta 4$-positive cell lines, ARRDC3 had only marginal effects on the parental, ITG $\beta 4$-negative MDA-MB-435 cell line (Supplementary Figures S4a-d). Altogether, these data show that ARRDC3 affects in vitro tumorigenicity, principally in an ITG $\beta 4$-dependent fashion. 

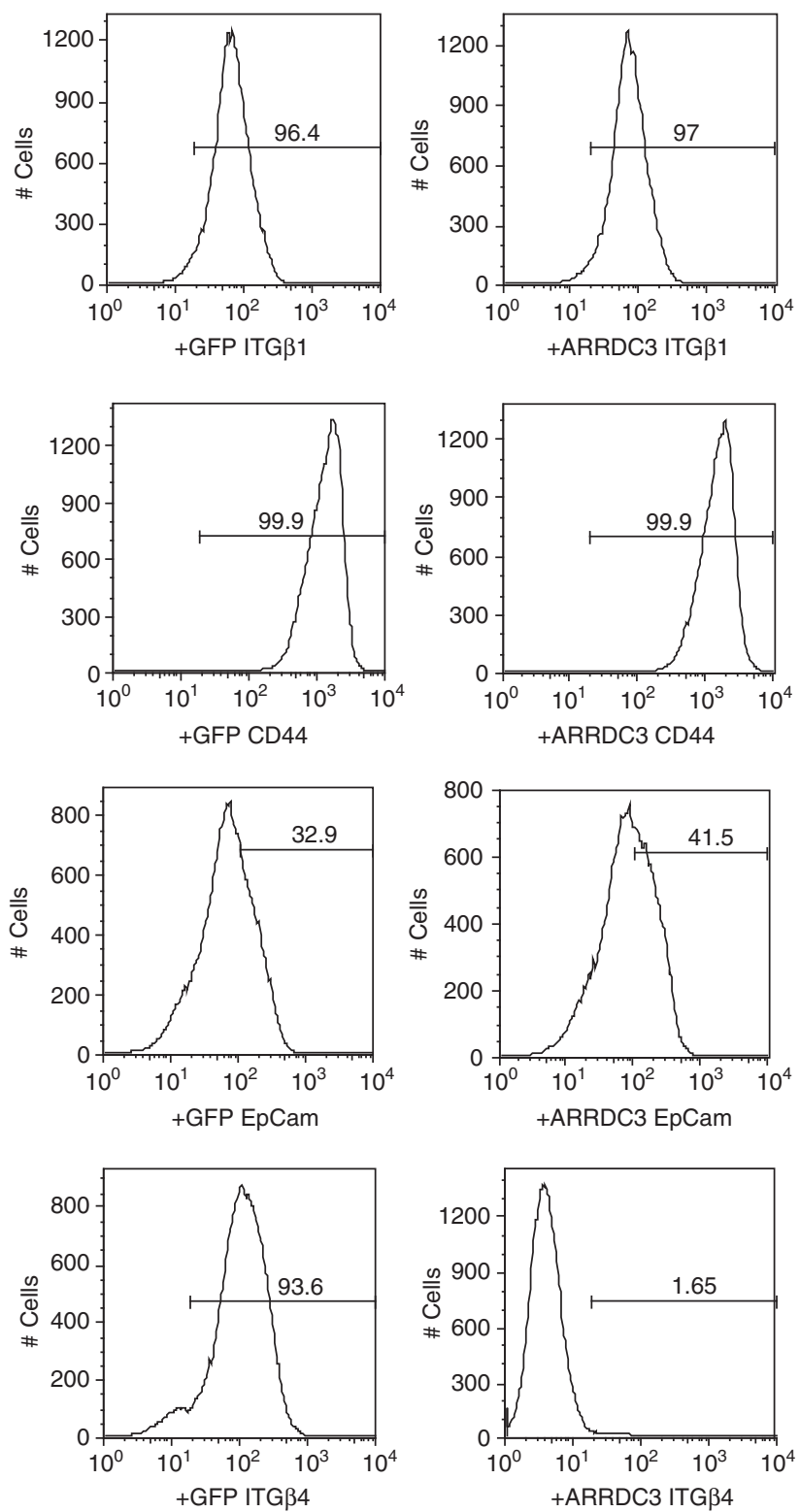

Figure 8 The regulation of ITG $\beta 4$ by ARRDC3 is specific and not a product of generalized increased endocytosis. MDA-MB-231 cells were infected with either a green fluorescent protein (GFP)expressing adenovirus ( + GFP) or an ARRDC3-expressing adenovirus ( + ARRDC3). Nonpermeabilized cells were then analyzed by flow cytometry for various cell surface proteins. ARRDC3 overexpression did not affect surface levels of ITG $\beta 1$, CD44 or EpCam.

\section{ARRDC3 downregulation and coordinate ITG $\beta 4$ upregulation in human breast carcinogenesis and tumor progression}

As there is an extensive connection between ITG $\beta 4$ and breast carcinogenesis, we examined protein levels of ARRDC3 and ITG $\beta 4$ in normal breast tissue and primary human breast cancers. ITG $\beta 4$-positive cells were located in the basal layer of normal ducts, whereas ARRDC3 was more highly expressed in luminal cells (in which ITG $\beta 4$ expression is negligible) but weakly expressed in basal cells (in which ITG $\beta 4$ expression is highest) (Figure 9b). In breast cancers, ARRDC3 expression was inversely correlated to ITG $\beta 4$ by immunofluorescence and western blot (Figures 9a and $\mathrm{c}-\mathrm{f}$ ).

The combination of our data and the current knowledge of ITG $\beta 4$ strongly suggested that ARRDC3 downregulation would lead to a more aggressive or metastatic phenotype. To analyze the expression pattern of ARRDC3 in tumors, 52 human breast tumors of varied grade were used for ITG $\beta 4$ and ARRDC3 coimmunofluorescence. Expression of ARRDC3 was inversely correlated to tumor grade (Table 1). Within grade 1 tumors samples, five out of six showed high ARRDC3 staining while the outlier exhibited strong ITG $\beta 4$ staining. Interestingly, ARRDC3 expression varied greatly in grade 2 tumors, yet levels were inversely correlated to ITG $\beta 4$ expression. Grade 3 tumors generally expressed low or undetectable levels of ARRDC3. Although 11 out of 52 tumors samples expressed no or low levels of ARRDC 3 and ITG $\beta 4$, only 1 out of 52 tumors samples had intense staining for both ARRCD3 and ITG $\beta 4$. Overall, the data support the hypothesis that ARRDC3 functions as a regulator of breast cancer progression through its affects on ITG $\beta 4$.

\section{Discussion}

In the present study, we describe a novel regulatory pathway involving the internalization and degradation of the cell surface protein ITG $\beta 4$, which has significant effects on breast cancer cell growth and tumorigenicity. We showed that ARRDC3, previously identified by genetic screening as a potential tumor suppressor (Adelaide et al., 2007), is a novel regulator of breast cancer progression. We show that ARRDC3 directly binds to ITG $\beta 4$, specifically when phosphorylated at S1424, ultimately leading to proteosome dependent degradation. The subsequent change in ITG $\beta 4$ protein levels significantly affects in vitro tumorigenic properties such as proliferation, migration, invasion and growth in soft agar. In vivo analyses show that ARRDC3 negatively regulates tumor growth in nude mice. In addition, data from human breast cancer samples show that ARRDC3 expression is inversely correlated to ITG $\beta 4$ protein levels and tumor grade.

ITG $\beta 4$ is part of a genetic signature correlated to basal-type breast cancer (Lu et al., 2008). It was therefore very interesting to note that ARRDC 3 is part of a cluster on chromosome 5 deleted in $17 \%$ of the same basal-type breast cancer subset (compared with $0 \%$ in luminal breast cancers) (Adelaide et al., 2007). Seven of eight basal-like breast cancer samples we analyzed had little to no expression of ARRDC3 (Table 1), suggesting that ARRDC3 may be downregulated by additional mechanisms other than chromosomal deletion. We found that when ARRDC3 protein was low or absent in human breast cancers regardless of grade or receptor status, these tumors generally had high levels of ITG $\beta 4$. We do not know the mechanism of downregulated ARRDC3 within these 

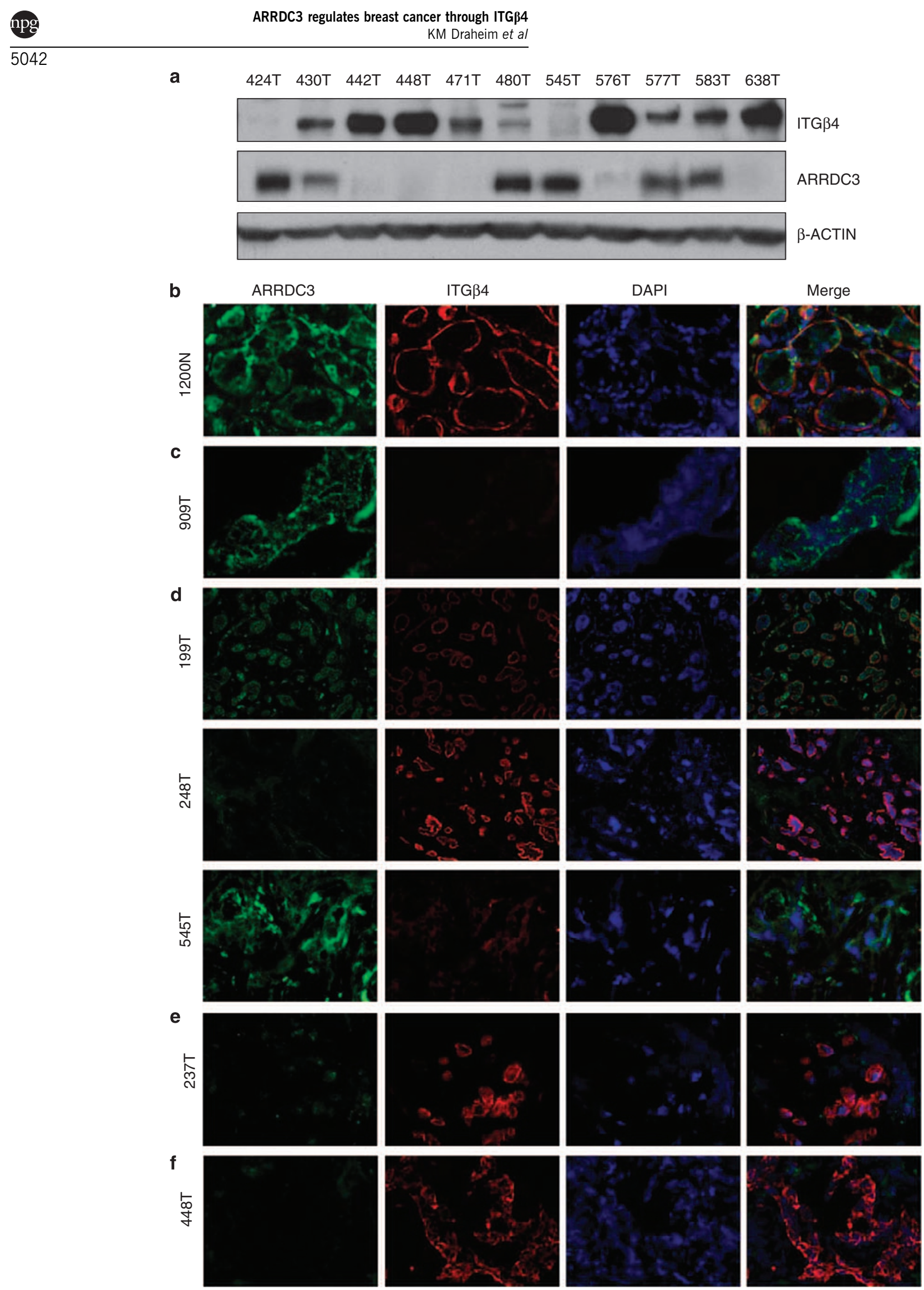
Table 1 Expression of ARRDC3 in human breast tumors

\begin{tabular}{|c|c|c|c|c|c|c|c|}
\hline$I D$ & $H E R 2$ & $E R$ & $P R$ & Cancer type & Grade & $I T G \beta 4$ & $A R R D C 3$ \\
\hline $0630 \mathrm{~T}$ & - & + & Weak & Lobular carcinoma & 1 & + & +++ \\
\hline $0832 \mathrm{~T}$ & - & + & - & Ductal carcinoma & 1 & + & +++ \\
\hline $0885 \mathrm{~T}$ & - & + & + & Ductal carcinoma & 1 & + & ++ \\
\hline 0909T & - & + & Weak & Ductal carcinoma & 1 & - & +++ \\
\hline $1334 \mathrm{~T}$ & - & + & + & Lobular carcinoma & 1 & +++ & + \\
\hline $1640 \mathrm{~T}$ & - & + & - & Ductal carcinoma & 1 & ++ & ++ \\
\hline 0199T & + & + & + & Ductal carcinoma & 2 & ++ & ++ \\
\hline 0248T & - & + & + & Ductal carcinoma & 2 & +++ & + \\
\hline $0327 \mathrm{~T}$ & + & + & + & Ductal carcinoma & 2 & ++ & ++ \\
\hline $0424 \mathrm{~T}$ & - & + & + & Papillary carcinoma & 2 & - & + \\
\hline 0448T & - & - & - & Ductal carcinoma & 2 & +++ & + \\
\hline $0462 \mathrm{~T}$ & - & + & - & Ductal carcinoma & 2 & ++ & ++ \\
\hline $0480 \mathrm{~T}$ & - & + & + & Ductal carcinoma & 2 & + & + \\
\hline $0545 \mathrm{~T}$ & + & + & - & Ductal carcinoma & 2 & ++ & ++ \\
\hline 0576T & - & + & - & Ductal carcinoma & 2 & +++ & + \\
\hline $0638 \mathrm{~T}$ & - & + & - & Ductal carcinoma & 2 & + & ++ \\
\hline $0837 \mathrm{~T}$ & Weak/- & - & - & Ductal carcinoma & 2 & ++ & + \\
\hline 0884T & - & + & - & Lobular carcinoma & 2 & ++ & + \\
\hline 0934T & + & - & - & Ductal carcinoma in situ & 2 & +++ & + \\
\hline 0960T & + & + & + & Ductal carcinoma & 2 & + & + \\
\hline $1003 \mathrm{~T}$ & - & + & + & Ductal carcinoma & 2 & - & ++ \\
\hline $1099 \mathrm{~T}$ & - & + & - & Ductal carcinoma & 2 & + & + \\
\hline $1281 \mathrm{~T}$ & - & + & + & Lobular carcinoma & 2 & ++ & - \\
\hline $1376 \mathrm{~T}$ & - & + & + & Lobular carcinoma & 2 & +++ & + \\
\hline $1403 \mathrm{~T}$ & + & + & + & Ductal carcinoma & 2 & ++ & ++ \\
\hline $1404 \mathrm{~T}$ & - & + & + & Lobular carcinoma & 2 & +++ & + \\
\hline $1471 \mathrm{~L}$ & - & + & + & Carcinoma & 2 & +++ & + \\
\hline $1471 \mathrm{~T}$ & - & + & + & Ductal carcinoma & 2 & +++ & + \\
\hline $1502 \mathrm{~T}$ & - & + & + & Ductal carcinoma & 2 & ++ & ++ \\
\hline $1569 \mathrm{~T}$ & - & + & + & Ductal carcinoma & 2 & + & +++ \\
\hline $0237 \mathrm{~T}$ & + & - & - & Ductal carcinoma & 3 & +++ & - \\
\hline 0316T & + & - & + & Ductal carcinoma & 3 & +++ & + \\
\hline $0442 \mathrm{~T}$ & + & - & - & Ductal carcinoma & 3 & +++ & + \\
\hline $0454 \mathrm{~T}$ & - & + & + & Lobular carcinoma & 3 & +++ & ++ \\
\hline $0471 \mathrm{~T}$ & + & + & - & Lobular carcinoma & 3 & - & + \\
\hline 0489T & - & + & + & Lobular carcinoma & 3 & + & - \\
\hline $0577 \mathrm{~T}$ & - & + & - & Lobular carcinoma & 3 & +++ & + \\
\hline 0690T & - & - & - & Ductal carcinoma & 3 & - & + \\
\hline $0738 \mathrm{~T}$ & - & - & + & Ductal carcinoma & 3 & +++ & + \\
\hline 0839T & - & - & - & Ductal carcinoma & 3 & - & - \\
\hline $0902 \mathrm{~T}$ & - & - & - & Ductal carcinoma & 3 & - & ++ \\
\hline $0957 \mathrm{~T}$ & - & - & - & Ductal carcinoma & 3 & +++ & + \\
\hline 0959M & - & + & - & Carcinoma & 3 & - & + \\
\hline $0959 \mathrm{~T}$ & - & + & - & Ductal carcinoma & 3 & + & + \\
\hline $1060 \mathrm{~T}$ & - & + & - & Ductal carcinoma & 3 & + & + \\
\hline $1097 \mathrm{~T}$ & Weak & + & Weak & Ductal carcinoma & 3 & ++ & + \\
\hline $1200 \mathrm{~T}$ & - & - & - & Ductal carcinoma & 3 & +++ & - \\
\hline $1342 \mathrm{M}$ & - & - & - & Ductal carcinoma & 3 & +++ & + \\
\hline $1550 \mathrm{~T}$ & - & + & + & Lobular carcinoma & 3 & +++ & + \\
\hline $1623 \mathrm{~T}$ & - & + & + & Ductal carcinoma & 3 & +++ & + \\
\hline $1645 \mathrm{~T}$ & NR & + & + & Lobular carcinoma & 3 & ++ & - \\
\hline $1659 \mathrm{~T}$ & - & + & - & Ductal carcinoma & 3 & +++ & + \\
\hline
\end{tabular}

Abbreviations: - , indicates no detectable expression; + , is low expression; ++ , is moderate expression; +++ , is high expression; ER, estrogen receptor; HER2, human epidermal growth factor receptor 2; ITG $\beta 4$, integrin $\beta 4$; NR, not reported; PR, progesterone receptor.

A table of the 52 human breast tumors analyzed for ARRDC3 and ITG $\beta 4$ expression using immunofluorescence. ID refers to the UMass Tissue Bank tumor number. HER2, ER and PR statuses, cancer type and grade are those determined at the time of diagnosis. All samples were processed at the same time using the same master-mix of primary and secondary antibodies. Images from each sample were taken all on the same day using the same exposure times for each channel. Expression levels were scored by a pathologist (SL) blinded to the sample information.

Figure 9 Expression of ARRDC3 in human breast tumors. (a) Western blot analysis of protein extracts from primary human breas tumors (invasive ductal carcinomas). ARRDC3 and ITG $\beta 4$ expression are inversely correlated. (b-f) Immunofluorescence of primary breast or breast tumor tissue demonstrated an inverse correlation to ARRDC3 and ITG $\beta 4$ protein levels. (b) ARRDC3 is primarily expressed in the luminal cells in normal breast tissue. (c) Grade 1 breast tumors tend to express high levels of ARRDC3 and low levels of ITG $\beta 4$. (d) Grade 2 human breast tumors showed varied expression levels of ARRDC3. Expression is always the inverse of ITG $\beta 4$ expression. (e) Grade 3 breast tumors tend to express low or undetectable levels of ARRDC 3 and high levels of ITG $\beta 4$. (f) Basal breast cancers with high ITG $\beta 4$ expression express very low or no levels of ARRDC3. 
tumors, however chromosome deletion is likely not the only cause of ARRDC3 defects. Further genetic, epigenetic and mutational analyses of the ARRDC3 locus are needed to more fully investigate the transcriptional regulation of ARRCD3 within tumors.

Interestingly, some tumors we evaluated maintained expression of both ARRDC3 and ITG $\beta 4$. It is possible that the effects of ARRDC3 on ITG $\beta 4$ are simply dose dependent and moderate expression of ARRDC3 maintains intermediate levels of ITG $\beta 4$. Low or moderate levels of ARRDC3 may also allow for the recycling of ITG $\beta 4$ similar to the effects of $\beta$-arrestin on GPCRs (Luttrell and Lefkowitz, 2002). The presence of increased surface ITG $\beta 4$ we observed after treatment with a proteosome inhibitor may be evidence of such a mechanism (Figure 5c). This would facilitate the speed in which filamentous actin protrusions form, thus promoting tumor progression. However, further trafficking studies are necessary to determine whether ITG $\beta 4$ is actively being recycled to the cell surface in the absence of degradation.

Until recently, there have been relatively few investigations of the factors involved in ITG endocytosis. Internalization of ITGs $\alpha 5 \beta 1, \alpha v \beta 6, \alpha v \beta 3, \alpha 6 \beta 1$ have been associated with clathrin-mediated endocytosis (Caswell and Norman, 2008; Ezratty et al., 2009), whereas ITGs $\alpha 5 \beta 1, \alpha v \beta 3, \alpha 2 \beta 1$ (Caswell and Norman, 2008; Shi and Sottile, 2008) have been connected to caveolae dependent endocytosis. ITG $\alpha 6 \beta 4$ has been shown to be associated with lipid rafts (GagnouxPalacios et al., 2003) suggesting that caveolae may have a role in localization. However, not all ITG $\alpha 6 \beta 4$ was found associated with lipid rafts. Our data provides additional insight to the area of ITG endocytosis. As ARRDC3 contains clathrin-binding motifs, it is possible that it functions within a clathrin-dependent mechanism although further investigation of the protein complexes within this pathway is needed. Lastly, ARRDC3 does not seem to affect $\beta 1 \mathrm{IGTs}$ (Figure 8), and because of the unique long cytoplasmic tail of ITG $\beta 4$, ARRDC3 is likely specific for this IGT.

It has been hypothesized that during the progression from normal epithelium to invasive carcinoma, the function of ITG $\beta 4$ switches from a mechanical adhesive device into a signaling-competent receptor. In this case, ITG $\beta 4$ needs to be liberated from hemidesmosome where it can then be trafficked to actin-rich motility structures (filopodia and lamellae) (Mercurio et al., 2001; Santoro et al., 2003; Lipscomb and Mercurio, 2005). Our findings highlight the importance of the ITG $\beta 4$-pS1424 site in this process as endogenous ARRDC3 colocalizes with this phosphorylated form of ITG $\beta 4$, likely during hemidesmosome disassembly and ITG $\beta 4$ internalization. The classic arrestin family member $\beta$-arrestin 1 is known to down-regulate G-protein mediated signaling, initiate internalization from the membrane and mediate additional signaling cascades (Claing et al., 2002). $\beta$-Arrestin 2 has been shown to have a similar role in controlling the single transmembrane receptor, transforming growth factor- $\beta$ R III (Chen et al., 2003). In addition, there is emerging evidence suggesting a role for $\beta$-arrestins in cancer (Buchanan et al., 2006; Raghuwanshi et al., 2008; Li et al., 2009). It is clear from our data that ARRDC3 has a role in the regulation of ITG $\beta 4$ protein levels and likely contributes to the control of ITG $\beta 4$ function during breast cancer progression.

Our data does not exclude the possibility that ARRDC3 has an ITG $\beta 4$ independent mechanism of action in cancer cells or in normal cells. The effects of ARRDC3 on cancer cells lacking ITG $\beta 4$ had marginal statistical significance and the overall effects were small (Supplementary Figures S2a-d). It is therefore unclear whether these effects are biologically significant. Considering its similarity to $\beta$-arrestins, ARRDC3 may also regulate GPCRs. Possible targets could include smoothened and frizzled receptors (class-6 GPCRs) or RhoA/C (class-1 GPCRs); which also have extensive implications in cancer.

In summary, our data identifies ARRDC3 as a novel regulator of breast cancer growth and progression that targets ITG $\beta 4$ for internalization and proteosome dependent degradation. We reveal several oncogenic properties affected by changes in ARRDC3 expression and identify a correlation between ARRDC3 expression and human breast tumor grade or aggressiveness. Our data also open several potential avenues of future research to better understand the potential significance of this novel regulatory pathway in carcinogenesis.

\section{Materials and methods}

\section{Cells and reagents}

MDA-MB-435 cancer cells expressing wild-type ITG $\beta 4$ were generated previously (Shaw et al., 1997). MDA-MB-231 breast carcinoma cells were obtained from the Lombardi Breast Cancer Depository (Georgetown University). The following antibodies were used: rabbit pAb 505 (ITG $\beta 4$-specific, gift from Dr Arthur Mercurio, UMass Medical School, Worcester, MA, USA); rat mAb 439-9B (ITG $\beta 4$-specific, BD Biosciences, San Jose, MA, USA); rabbit pAb S1424 (ITG $\beta 4$ phosphoserine 1424 specific, gift from Dr Isaac Rabinovitz, BIDMC, Boston, MA, USA); rabbit pAb ARRDC3 (Abcam, Cambridge, MA, USA); and mouse mAb Arrdc3 generated in our laboratory.

\section{Cloning and generation of stable lines}

pSuper vectors (oligoengine) containing control short hairpin RNA (shCtl target sequence: TTCTCCGAACGTGTCA CGT) and short hairpin RNAs targeting ARRDC3 (target sequence A:GGCCTTGGCTACTACCAGT; target sequence C: GCGTGGAATATTCACTAAT) were generated in our lab per manufacturers recommended protocol. Full-length human ARRDC3 cDNA and Flag-LacZ fusion cDNA were cloned into the pBABE-puro expression vector (Addgene, Cambridge, MA, USA). Cells were transfected with Fugene HD (Roche Applied Science, Indianapolis, IN, USA) following manufacturer's protocol. Stable transformants were selected using puromycin and pooled.

\section{Adenoviral preparation}

Full-length human ARRDC3 cDNA was initially cloned into pLE green fluorescent protein (Clontech, Mountain View, CA, 
USA). The fragment containing ARRDC3 + green fluorescent protein was then cloned into Adeno-X LP CMV using the Adeno-X Expression System 2 kit (Clontech). Adeno-X maxi purification kit (Clontech) was used to purify the adenovirus. For all steps, the manufacturer's protocol was followed. The amount of virus needed for each cell type was determined empirically.

\section{Immunofluorescence}

Cells cultured on coverslips or frozen sections of O.C.T.-fixed human breast cancer tissue (obtained from the UMass Cancer Center Tumor Bank with IRB approval \#13176) were fixed with acetone and permeabilized by incubating with phosphatebuffered saline + Triton and blocked with $5 \%$ goat serum (Gibco, Carlsbad, CA, USA). Slides were incubated with primary antibody (rabbit polyclonal ARRDC3 (Abcam, Cambridge, MA, USA) and/or ITG $\beta 4$ 439-9b (BD-Pharmingen, San Jose, CA, USA)) for $2 \mathrm{~h}$ at room temperature or overnight at $4{ }^{\circ} \mathrm{C}$. After washing, slides were incubated with secondary antibodies conjugated with either fluorescein isothiocyanate or Texas-Red (Vector Laboratories, Burlingame, CA, USA) for $2 \mathrm{~h}$ (room temp). Slides were mounted using Vectashield with 4'-6-diamidino-2-phenylindole (Vector Laboratories) and were analyzed using a fluorescence microscope.

\section{Wound assay}

In all, $5 \times 10^{5}$ cells were evenly plated in a six-well plate. At $24 \mathrm{~h}$ after plating, confluent cells were treated with $15 \mu \mathrm{g} / \mathrm{ml}$ mitomycin C (Roche Applied Science) for $90 \mathrm{~min}$ at $37^{\circ} \mathrm{C}$. The monolayer was scratched with a P200 pipette tip and washed three times to remove floating cells. The wound closure was then monitored by digital photography.

\section{Growth in soft agar}

In all, $1.0 \times 10^{3}$ cells were suspended in $2 \mathrm{ml}$ of serumcontaining medium containing $0.3 \%$ agar and overlaid on a $1 \mathrm{ml}$ base layer of $0.75 \%$ agar in six-well plates. The soft agar was overlaid with complete medium $(0.5 \mathrm{ml}$ per well), which was changed every 2 days. After 3-4 weeks of incubation, viable colonies were stained by adding 3-(4,5-dimethylthiazol2-yl)-2,5-diphenyltetrazolium bromide into the covering media. Images were captured using bright-field microscopy and the number and size of the colonies were analyzed using ImageJ software. Only colonies with a diameter of $>50 \mu \mathrm{m}$ were counted.

\section{Invasion}

Matrigel invasion assays were carried out as described previously using $6.5-\mathrm{mm}$ Transwell chambers $(8-\mu \mathrm{m}$ pore size, CoStar, St Louis, MO, USA) (Shaw et al., 1997). After $4 \mathrm{~h}$, the cells that had invaded to the lower surface of the filters were fixed in methanol for $10 \mathrm{~min}$. Filters were mounted on glass slides using Vectashield mounting medium containing 4'-6-diamidino-2-phenylindole (Vector Laboratories). Invasion was quantified by counting the number of stained nuclei in five independent fields in each transwell using ImageJ software.

\section{Xenograft mouse studies}

Animals' care was in accordance with guidelines approved by IACUC. Cells were trypsinized, washed with sterile phosphatebuffered saline and resuspended in $35 \mu \mathrm{l}$ phenol red-free Matrigel immediately before injection. Old female immunocompromised mice ( 9 weeks) $(n u / n u$; National Cancer In- stitute) were anesthetized briefly with isofluorane and cells were injected into the \#3 and \#8 mammary fat pad $\left(1 \times 10^{6}\right.$ cells per injection, two injection sites per mouse). Tumor volume was determined using the following formula: $(4 / 3) \pi(1 /$

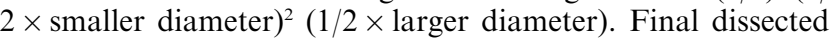
tumor volume was determined using the following formula: $(4 / 3)$ $\pi(1 / 2 \times$ length $)(1 / 2 \times$ width $)(1 / 2 \times$ height $)$. Percent tumor necrosis was determined by outlining the necrotic area in the area finder function of ImageJ and dividing it by the total area of the tumor.

\section{Immunoprecipitation}

Cells were treated for 3-4h with $1 \mathrm{~mm}$ sodium orthovanadate or $1 \mathrm{~mm} \mathrm{NaF}$ or $50 \mu \mathrm{M}$ Lactacystin and solubilized at $4{ }^{\circ} \mathrm{C}$ for $15 \mathrm{~min}$ in NP-40 Lysis buffer supplemented with $1 \mathrm{~mm}$ sodium orthovanadate, $1 \mathrm{~mm} \mathrm{NaF}$ and protease inhibitors (Complete mini tab; Roche Applied Science). Aliquots of cell extracts containing equivalent protein amounts were incubated overnight at $4{ }^{\circ} \mathrm{C}$ with antibodies and protein-A/G-Sepharose (Santa Cruz, Santa Cruz, CA, USA) with constant agitation. Immune complexes and aliquots of cell extracts containing equivalent total protein amounts were then analyzed by western blot.

\section{Western blot analysi.}

Cell extracts containing equivalent protein amounts were resolved by sodium dodecyl sulfate-polyacrylamide gel and were transferred to nitrocellulose membranes. Membranes were blocked for an hour using a $50 \mathrm{~mm}$ Tris buffer $(\mathrm{pH} 7.5)$ containing $0.15 \mathrm{~mm} \mathrm{NaCl}$ and $0.05 \%$ Tween 20 and $5 \%(\mathrm{w} / \mathrm{v})$ Blocker (BioRad, Hercules, CA, USA) in phosphate-buffered saline + Tween 20. Membranes were incubated overnight at $4{ }^{\circ} \mathrm{C}$ in the same buffer containing primary antibodies. Proteins were detected by enhanced chemiluminescence (Pierce, Rockford, IL, USA). For phospho-immunoblots, the blocking buffer for the primary antibodies contained $5 \%(\mathrm{w} / \mathrm{v})$ bovine serum albumin.

\section{Statistical analysis}

All values in the present study were expressed as mean \pm s.e.m. unless otherwise noted. The significant differences between the groups were analyzed by a Student's $t$-test and a $P$-value of $<0.05$ was considered significant.

\section{Abbreviations}

EpCam, epithelial cell adhesion molecule; ER, estrogen receptor; GPCRs, G-protein coupled receptors; HD, hemidesmosomes; ITG $\beta 1$, integrin $\beta 1$; ITG $\beta 4$, integrin $\beta 4$; PKC, protein kinase $\mathrm{C}$; $\mathrm{PR}$, progesterone receptor; OCT, optimal cutting temperature compound.

\section{Conflict of interest}

The authors declare no conflict of interest.

\section{Acknowledgements}

We thank Shannon Pankratz, Yulian Ramirez and Carolyn Padden for their help with the xenograft studies. We appreciate the help from the UMass Cancer Center Tissue Bank (http:// www.umassmed.edu/cancercenter/tissuebank/index.aspx). 
Funding: this work was supported by the National Cancer Institute of the National Institutes of Health (grant number CA118916) and The Worcester Foundation for Biotechnology Research (SRL).

\section{References}

Adelaide J, Finetti P, Bekhouche I, Repellini L, Geneix J, Sircoulomb $\mathrm{F}$ et al. (2007). Integrated profiling of basal and luminal breast cancers. Cancer Res 67: 11565-11575.

Alvarez CE. (2008). On the origins of arrestin and rhodopsin. BMC Evol Biol 8: 222.

Arnaout MA, Mahalingam B, Xiong JP. (2005). Integrin structure, allostery, and bidirectional signaling. Annu Rev Cell Dev Biol 21: $381-410$

Aubry L, Guetta D, Klein G. (2009). The arrestin fold: variations on a theme. Curr Genomics 10: 133-142.

Baril P, Gangeswaran R, Mahon PC, Caulee K, Kocher HM, Harada $\mathrm{T}$ et al. (2007). Periostin promotes invasiveness and resistance of pancreatic cancer cells to hypoxia-induced cell death: role of the beta4 integrin and the PI3k pathway. Oncogene 26: 2082-2094.

Bhandari D, Trejo J, Benovic JL, Marchese A. (2007). Arrestin-2 interacts with the ubiquitin-protein isopeptide ligase atrophininteracting protein 4 and mediates endosomal sorting of the chemokine receptor CXCR4. J Biol Chem 282: 36971-36979.

Buchanan FG, Gorden DL, Matta P, Shi Q, Matrisian LM, DuBois RN. (2006). Role of beta-arrestin 1 in the metastatic progression of colorectal cancer. Proc Natl Acad Sci USA 31: 1492-1497.

Caswell P, Norman J. (2008). Endocytic transport of integrins during cell migration and invasion. Trends Cell Biol 18: 257-263.

Caswell PT, Norman JC. (2006). Integrin trafficking and the control of cell migration. Traffic 7: 14-21.

Chen M, Sinha M, Luxon BA, Bresnick AR, O'Connor KL. (2009). Integrin alpha6beta4 controls the expression of genes associated with cell motility, invasion, and metastasis, including S100A4/ metastasin. J Biol Chem 284: 1484-1494.

Chen W, Kirkbride KC, How T, Nelson CD, Mo J, Frederick JP et al. (2003). Beta-Arrestin 2 mediates endocytosis of type III TGF-beta receptor and down-regulation of its signaling. Science 301: 1394-1397.

Claing A, Laporte SA, Caron MG, Lefkowitz RJ. (2002). Endocytosis of $\mathrm{G}$ protein-coupled receptors: roles of $\mathrm{G}$ proteincoupled receptor kinases and beta-arrestin proteins. Prog Neurobiol 66: 61-79.

Diaz LK, Cristofanilli M, Zhou X, Welch KL, Smith TL, Yang Y et al. (2005). Beta4 integrin subunit gene expression correlates with tumor size and nuclear grade in early breast cancer. Mod Pathol 18: $1165-1175$.

Dowling J, Yu QC, Fuchs E. (1996). Beta4 integrin is required for hemidesmosome formation, cell adhesion and cell survival. $J$ Cell Biol 134: 559-572.

Dutta U, Shaw LM. (2008). A key tyrosine (Y1494) in the beta4 integrin regulates multiple signaling pathways important for tumor development and progression. Cancer Res 68: 8779-8787.

Ezratty EJ, Bertaux C, Marcantonio EE, Gundersen GG. (2009). Clathrin mediates integrin endocytosis for focal adhesion disassembly in migrating cells. $J$ Cell Biol 187: 733-747.

Falcioni R, Perrotti N, Piaggio G, Kennel SK, Sacchi A. (1989). Insulin-induced phosphorylation of the beta-4 integrin subunit expressed on murine metastatic carcinoma cells. Mol Carcinog 2: 361-368.

Fuchs E, Dowling J, Segre J, Lo SH, Yu QC. (1997). Integrators of epidermal growth and differentiation: distinct functions for beta 1 and beta 4 integrins. Curr Opin Genet Dev 7: 672-682.

Gagnoux-Palacios L, Dans M, van't Hof W, Mariotti A, Pepe A, Meneguzzi G et al. (2003). Compartmentalization of integrin \{alpha\}6\{beta\}4 signaling in lipid rafts. J Cell Biol 162: 11891196.
Author Contributions: The experiments were designed and performed by KMD, HC, QT, NM, MR and SL. The paper was written by KMD and SL The principle investigator is SL.
Germain EC, Santos TM, Rabinovitz I. (2009). Phosphorylation of a novel site on the $\{$ beta 44 integrin at the trailing edge of migrating cells promo. Mol Biol Cell 20: 56-67.

Gurevich EV, Gurevich VV. (2006). Arrestins: ubiquitous regulators of cellular signaling pathways. Genome Biol 7: 236.

Hemler ME. (2001). Retooling of the beta 4 integrin in tumor cellsligands lost and kinase gained. Dev Cell 1: 728-730.

Hynes RO. (2002). Integrins: bidirectional, allosteric signaling machines. Cell 110: 673-687.

Kendall RT, Luttrell LM. (2009). Diversity in arrestin function. Cell Mol Life Sci 66: 2953-2973.

Lee EC, Lotz MM, Steele Jr GD, Mercurio AM. (1992). The integrin alpha 6 beta 4 is a laminin receptor. $J$ Cell Biol 117: 671-678.

Li TT, Alemayehu M, Aziziyeh AI, Pape C, Pampillo M, Postovit LM et al. (2009). Beta-arrestin/Ral signaling regulates lysophosphatidic acid-mediated migration and invasion of human breast tumor cells. Mol Cancer Res 7: 1064-1077.

Lipscomb EA, Dugan AS, Rabinovitz I, Mercurio AM. (2003). Use of RNA interference to inhibit integrin (alpha6beta4)-mediated invasion and migration of breast carcinoma cells. Clin Exp Metastasis 20: $569-576$.

Lipscomb EA, Mercurio AM. (2005). Mobilization and activation of a signaling competent alpha6beta4integrin underlies its contribution to carcinoma progression. Cancer Metastasis Rev 24: 413-423.

Lipscomb EA, Simpson KJ, Lyle SR, Ring JE, Dugan AS, Mercurio AM. (2005). The alpha6beta4 integrin maintains the survival of human breast carcinoma cells in vivo. Cancer Res $\mathbf{6 5}$ : 10970-10976.

Lu S, Simin K, Khan A, Mercurio AM. (2008). Analysis of integrin beta4 expression in human breast cancer: association with basal-like tumors and prognostic significance. Clin Cancer Res 14: 1050-1058.

Luttrell LM, Lefkowitz RJ. (2002). The role of beta-arrestins in the termination and transduction of G-protein-coupled receptor signals. J Cell Sci 115: 455-465.

Mercurio AM, Rabinovitz I, Shaw LM. (2001). The alpha 6 beta 4 integrin and epithelial cell migration. Curr Opin Cell Biol 13: $541-545$.

Mitra SK, Schlaepfer DD. (2006). Integrin-regulated FAK-Src signaling in normal and cancer cells. Curr Opin Cell Biol 18: 516-523.

Mosesson Y, Mills GB, Yarden Y. (2008). Derailed endocytosis: an emerging feature of cancer. Nat Rev Cancer 8: 835-850.

Nikolopoulos SN, Blaikie P, Yoshioka T, Guo W, Giancotti FG. (2004). Integrin beta4 signaling promotes tumor angiogenesis. Cancer Cell 6: 471-483.

O'Connor KL, Shaw LM, Mercurio AM. (1998). Release of cAMP gating by the alpha6beta4 integrin stimulates lamellae formation and the chemotactic migration of invasive carcinoma cells. $J$ Cell Biol 143: 1749-1760.

Oka S, Masutani H, Liu W, Horita H, Wang D, Kizaka-Kondoh S et al. (2006). Thioredoxin-binding protein-2-like inducible membrane protein is a novel vitamin D3 and peroxisome proliferatoractivated receptor (PPAR)gamma ligand target protein that regulates PPARgamma signaling. Endocrinology 147: 733-743.

Perou CM, Sorlie T, Eisen MB, van de Rijn M, Jeffrey SS, Rees CA et al. (2000). Molecular portraits of human breast tumours. Nature 406: $747-752$.

Rabinovitz I, Mercurio AM. (1996). The integrin alpha 6 beta 4 and the biology of carcinoma. Biochem Cell Biol 74: 811-821.

Rabinovitz I, Tsomo L, Mercurio AM. (2004). Protein kinase C-alpha phosphorylation of specific serines in the connecting segment of the 
beta 4 integrin regulates the dynamics of type II hemidesmosomes. Mol Cell Biol 24: 4351-4360.

Raghuwanshi SK, Nasser MW, Chen X, Strieter RM, Richardson RM. (2008). Depletion of beta-arrestin-2 promotes tumor growth and angiogenesis in a murine model of lung cancer. $J$ Immunol 180: 5699-5706.

Rakha EA, Ellis IO. (2009). Triple-negative/basal-like breast cancer: review. Pathology 41: 40-47.

Rakha EA, Reis-Filho JS, Ellis IO. (2008). Basal-like breast cancer: a critical review. J Clin Oncol 26: 2568-2581.

Raymond K, Kreft M, Song JY, Janssen H, Sonnenberg A. (2007). Dual Role of alpha6beta4 integrin in epidermal tumor growth: tumor-suppressive versus tumor-promoting function. $\mathrm{Mol}$ Biol Cell 18: $4210-4221$

Santoro MM, Gaudino G, Marchisio PC. (2003). The MSP receptor regulates alpha6beta4 and alpha3beta1 integrins via 14-3-3 proteins in keratinocyte migration. Dev Cell 5: 257-271.

Sørlie T, Perou CM, Tibshirani R, Aas T, Geisler S, Johnsen H et al. (2001). Gene expression patterns of breast carcinomas distinguish tumor subclasses with clinical implications. Proc Natl Acad Sci USA 98: 10869-10874.

Shaw LM, Rabinovitz I, Wang HH, Toker A, Mercurio AM. (1997). Activation of phosphoinositide 3-OH kinase by the alpha6beta4 integrin promotes carcinoma invasion. Cell 91: 949-960.

Shi F, Sottile J. (2008). Caveolin-1-dependent $\{$ beta $\} 1$ integrin endocytosis is a critical regulator of fibronectin turnove. $\mathrm{J} \mathrm{Cell} \mathrm{Sci}$ 121: $2360-2371$.

Sotiriou C, Pusztai L. (2009). Gene-expression signatures in breast cancer. $N$ Engl J Med 360: 790-800.
Vicente-Manzanares M, Choi CK, Horwitz AR. (2009). Integrins in cell migration - the actin connection. $J$ Cell Sci 122: 199-206.

Voduc D, Nielsen TO. (2008). Basal and triple-negative breast cancers: impact on clinical decision-making and novel therapeutic options. Clin Breast Cancer 8(Suppl 4): S171-S178.

Watt FM. (2002). Role of integrins in regulating epidermal adhesion, growth and differentiation. EMBO J 21: 3919-3926.

Wilhelmsen $\mathrm{K}$, Litjens SH, Kuikman I, Margadant C, van Rheenen J, Sonnenberg A. (2007). Serine phosphorylation of the integrin beta4 subunit is necessary for epidermal growth factor receptor induced hemidesmosome disruption. Mol Biol Cell 18: 3512-3522.

Wilhelmsen K, Litjens SHM, Sonnenberg A. (2006). Multiple Functions of the integrin $\{$ alpha 6 6 beta $\} 4$ in epidermal homeostasis and tumorigenesis. Mol Cell Biol 26: 2877-2886.

Yoon SO, Shin S, Mercurio AM. (2005). Hypoxia stimulates carcinoma invasion by stabilizing microtubules and promoting the Rab11 trafficking of the alpha6beta4 integrin. Cancer Res 65: 2761-2769.

Zhang Z, Hao J, Zhao Z, Ben P, Fang F, Shi L et al. (2009). beta]Arrestins facilitate ubiquitin-dependent degradation of apoptosis signal-regulating kinase 1 (ASK1) and attenuate H2O2-induced apoptosis. Cellular Signalling 21: 1195-1206.

(c) This work is licensed under the Creative Commons Attribution-NonCommercial-Share Alike 3.0 Unported License. To view a copy of this license, visit http://creativecommons.org/licenses/by-nc-sa/3.0/

Supplementary Information accompanies the paper on the Oncogene website (http://www.nature.com/onc) 